\title{
Higher-Order Hermite-Fejér Interpolation for Stieltjes Polynomials
}

\author{
Hee Sun Jung ${ }^{1}$ and Ryozi Sakai ${ }^{2}$ \\ ${ }^{1}$ Department of Mathematics Education, Sungkyunkwan University, Seoul 110-745, Republic of Korea \\ ${ }^{2}$ Department of Mathematics, Meijo University, Nagoya 468-8502, Japan
}

Correspondence should be addressed to Hee Sun Jung; hsun90@skku.edu

Received 5 July 2013; Accepted 22 August 2013

Academic Editor: Qiankun Song

Copyright (c) 2013 H. S. Jung and R. Sakai. This is an open access article distributed under the Creative Commons Attribution License, which permits unrestricted use, distribution, and reproduction in any medium, provided the original work is properly cited.

Let $w_{\lambda}(x):=\left(1-x^{2}\right)^{\lambda-1 / 2}$ and $P_{\lambda, n}$ be the ultraspherical polynomials with respect to $w_{\lambda}(x)$. Then, we denote the Stieltjes polynomials $E_{\lambda, n+1}$ with respect to $w_{\lambda}(x)$ satisfying $\int_{-1}^{1} w_{\lambda}(x) P_{\lambda, n}(x) E_{\lambda, n+1}(x) x^{m} d x(=0,0 \leq m<n+1 ; \neq 0, m=n+1)$. In this paper, we consider the higher-order Hermite-Fejér interpolation operator $H_{n+1, m}$ based on the zeros of $E_{\lambda, n+1}$ and the higher order extended Hermite-Fejér interpolation operator $\mathscr{H}_{2 n+1, m}$ based on the zeros of $E_{\lambda, n+1} P_{\lambda, n}$. When $m$ is even, we show that Lebesgue constants of these interpolation operators are $O\left(n^{\max \{(1-\lambda) m-2,0\}}\right)(0<\lambda<1)$ and $O\left(n^{\max \{(1-2 \lambda) m-2,0\}}\right)(0<\lambda<1 / 2)$, respectively; that is, $\left\|\mathscr{H}_{2 n+1, m}\right\|=O\left(n^{\max \{(1-2 \lambda) m-2,0\}}\right)(0<\lambda<1)$ and $\left\|H_{n+1, m}\right\|=O\left(n^{\max \{(1-\lambda) m-2,0\}}\right)(0<\lambda<1 / 2)$. In the case of the Hermite-Fejér interpolation polynomials $\mathscr{H}_{2 n+1, m}[\cdot]$ for $1 / 2 \leq \lambda<1$, we can prove the weighted uniform convergence. In addition, when $m$ is odd, we will show that these interpolations diverge for a certain continuous function on $[-1,1]$, proving that Lebesgue constants of these interpolation operators are similar or greater than $\log n$.

\section{Introduction}

Let $X:=\left\{x_{k, n}\right\} \subset[-1,1]$ and

$$
\begin{array}{r}
-1<x_{1, n}<x_{2, n}<\cdots<x_{n-1, n}<x_{n, n}<1, \\
n=1,2, \ldots .
\end{array}
$$

For any real-valued function $f$ on $[-1,1]$ and an integer $m \geq 1$, we recall that there exist unique Hermite and HermiteFejér interpolatory polynomials of higher order denoted by $H_{n, m}(f, X)$, and of degree $\leq n m-1$, defined as follows:

$$
\begin{aligned}
& H_{n, m}\left(f, X, x_{k, n}\right)=f\left(x_{k, n}\right), \quad 1 \leq k \leq n ; \\
& H_{n, m}^{(t)}\left(f, X, x_{k, n}\right)=0, \quad 1 \leq t \leq m-1,
\end{aligned}
$$

$$
1 \leq k \leq n
$$

We note that, by definition, $H_{n, 1}$ is the Lagrange, $H_{n, 2}$ is the Hermite-Fejér, and $H_{n, 4}$ is the Krylov-Stayermann interpolatory polynomial. By (2), we may write

$$
H_{n, m}(f, X, x)=\sum_{k=1}^{n} f\left(x_{k, n}\right) h_{k, n, m}(X, x),
$$

$$
n=1,2, \ldots
$$

The polynomials

$$
h_{k, n, m}(X, x)=l_{k, n}^{m}(X, x) \sum_{i=0}^{m-1} e_{i, k, n, m}\left(x-x_{k, n}\right)^{i},
$$


are unique, of degree exactly $n m-1$ and satisfy the relations

$$
\begin{array}{r}
h_{k, n, m}^{(t)}\left(X, x_{l, n}\right)=\delta_{0, t} \delta_{l, k}, \quad 1 \leq k, l \leq n, \\
0 \leq t \leq m-1,
\end{array}
$$

where for nonnegative integers $u$ and $v$

$$
\delta_{u, v}:= \begin{cases}1, & u=v \\ 0, & u \neq v\end{cases}
$$

Here, $l_{k, n}(X, x)$ are the well-known fundamental Lagrange polynomials of degree $n-1$ given by

$$
\begin{aligned}
l_{k, n}(X, x) & :=\frac{w_{n}(x)}{w_{n}^{\prime}\left(x_{k, n}\right)\left(x-x_{k, n}\right)}, \\
w_{n}(x) & :=\prod_{k=1}^{n}\left(x-x_{k, n}\right),
\end{aligned}
$$

and the coefficients $e_{i, k}$ may be obtained from the relations

$$
\begin{array}{rr}
h_{k, n, m}\left(X, x_{l, n}\right)=\delta_{l, k}, & 1 \leq k, l \leq n ; \\
h_{k, n, m}^{(t)}\left(X, x_{l, n}\right)=0, & 1 \leq t \leq m-1, \\
& 1 \leq k, l \leq n .
\end{array}
$$

If $f \in C^{(m-1)}[-1,1]$, then the Hermite interpolation polynomial $\widehat{H}_{n, m}(f, X, x)$ of degree $\leq n m-1$ with respect to $X$ is defined by

$$
\begin{array}{r}
\widehat{H}_{n, m}^{(t)}\left(f, X, x_{k, n}\right):=f^{(t)}\left(x_{k, n}\right) \\
1 \leq k \leq n, 0 \leq t \leq m-1 .
\end{array}
$$

We may express $\widehat{H}_{n, m}(f, X, x)$ as

$$
\begin{array}{r}
\widehat{H}_{n, m}(f, X, x)=\sum_{t=0}^{m-1} \sum_{k=1}^{n} f^{(t)}\left(x_{k, n}\right) h_{t, k, n, m}(X, x), \\
m=1,2, \ldots,
\end{array}
$$

where for $0 \leq t \leq m-1$

$$
\begin{aligned}
& h_{t, k, n, m}(X, x) \\
& \quad=l_{k, n}^{m}(X, x) \frac{\left(x-x_{k, n}\right)^{t}}{t !} \sum_{i=0}^{m-1-t} e_{t, i, k, n, m}\left(x-x_{k, n}\right)^{i}
\end{aligned}
$$

is the unique polynomial of degree $n m-1$ satisfying

$$
\begin{array}{r}
h_{t, k, n, m}^{(i)}\left(X, x_{j, n}\right)=\delta_{t, i} \delta_{k, j}, \quad 0 \leq i, t \leq m-1, \\
1 \leq j, k \leq n .
\end{array}
$$

Then, we easily see from the relations (5) and (12) that $h_{0, k, n, m}(x)=h_{k, n, m}(x), e_{0, i, k, n, m}=e_{i, k, n, m}$, and $e_{t, i, k, n, m}=$ $e_{0, i, k, n, m}$ for $1 \leq k \leq n$ and $0 \leq i, t \leq m-1$ (see [1]). Now, we have for any polynomial $P$ of degree $\leq n m-1$,

$$
\begin{aligned}
P(x) & =\widehat{H}_{n, m}(P, X, x) \\
& =H_{n, m}(P, X, x)+\sum_{t=1}^{m-1} \sum_{k=1}^{n} P^{(t)}\left(x_{k, n}\right) h_{t, k, n, m}(X, x) .
\end{aligned}
$$

In what follows, we abbreviate several notations as $h_{k}(x):=$ $h_{k, n, m}(x), e_{i, k}:=e_{i, k, n, m}$, and $e_{t, i, k}:=e_{t, i, k, n, m}$ if there is no confusion. Here, we are interested in Hermite-Fejér and Hermite interpolations with respect to $X$ whose elements are the zeros of a sequence of Stieltjes polynomials and the product polynomials of Stieltjes polynomials and the ultraspherical polynomials, respectively. To be precise, we first consider the generalized Stieltjes polynomials $E_{\lambda, n+1}(x)$ defined (up to a multiplicative constant) by

$$
\begin{array}{r}
\int_{-1}^{1} w_{\lambda}(x) P_{\lambda, n}(x) E_{\lambda, n+1}(x) x^{k} d x=0, \\
k=0,1,2, \ldots, n, n \geq 1,
\end{array}
$$

where $w_{\lambda}(x)=\left(1-x^{2}\right)^{\lambda-1 / 2}, \lambda>-1 / 2$, and $P_{\lambda, n}(x)$ is the $n$th ultraspherical polynomial for the weight function $w_{\lambda}(x)$. In 1935, Szegö [2] showed that the zeros of the generalized Stieltjes polynomials $E_{\lambda, n+1}(x)$ are real and inside $[-1,1]$ and interlace with the zeros of $P_{\lambda, n}(x)$ whenever $0 \leq \lambda \leq 2$.

For the properties of interpolation operators based at the zeros of $E_{\lambda, n+1}$ and the zeros of $P_{\lambda, n} E_{\lambda, n+1}$, Ehrich and Mastroianni [3, 4] proved that Lagrange interpolation operators $L_{n+1}$ based on the zeros of $E_{\lambda, n+1}$ and extended Lagrange interpolation operators $\mathscr{L}_{2 n+1}$ based on the zeros of $E_{\lambda, n+1} P_{\lambda, n}$ have Lebesgue constants $\left\|L_{n+1}\right\|_{\infty}(0<\lambda<1)$ and $\left\|\mathscr{L}_{2 n+1}\right\|_{\infty}(0<\lambda \leq 1 / 2)$ of optimal order, that is, $O(\log n)$. For the Hermite-Fejér interpolation operator $H_{n+1}$ based on the zeros of $E_{\lambda, n+1}$ and the extended Hermite-Fejér interpolation operator $\mathscr{H}_{2 n+1}$ based on the zeros of $E_{\lambda, n+1} P_{\lambda, n}$, it is proved that Lebesgue constants $\left\|H_{n+1}\right\|_{\infty}(0<\lambda<1)$ and $\left\|\mathscr{H}_{2 n+1}\right\|_{\infty}(0<\lambda \leq 1 / 2)$ are of optimal order, that is, $O(1)$, in [5]. In this paper, we consider the higher-order Hermite-Fejér interpolation operator $H_{n+1, m}$ based on the zeros of $E_{\lambda, n+1}$ and the higher-order extended Hermite-Fejér interpolation operator $\mathscr{H}_{2 n+1, m}$ based on the zeros of $E_{\lambda, n+1} P_{\lambda, n}$. When $m$ is even, we show that Lebesgue constants of these interpolation operators are $O\left(n^{\max \{(1-\lambda) m-2,0\}}\right)$ and $O\left(n^{\max \{(1-2 \lambda) m-2,0\}}\right)$, respectively; that is, $\left\|H_{n+1, m}\right\|=O\left(n^{\max \{(1-\lambda) m-2,0\}}\right)(0<\lambda<1)$ and $\left\|\mathscr{H}_{2 n+1, m}\right\|$ $=O\left(n^{\max \{(1-2 \lambda) m-2,0\}}\right)(0<\lambda<1 / 2)$. In the case of the Hermite-Fejér interpolation polynomials $\mathscr{H}_{2 n+1, m}[\cdot]$ for $1 / 2 \leq \lambda<1$, we can prove the weighted uniformconvergence. In addition, when $m$ is odd, we will show that these interpolations diverge for a certain continuous function on $[-1,1]$, proving that Lebesgue constants of these interpolation operators are similar or greater than $\log n$. 
This paper is organized as follows. In Section 2, we will introduce the main results. In Section 3, we will show the auxiliary propositions and estimate the coefficients of Hermite-Fejér interpolation polynomials in order to prove the main results. Finally, we will prove the results in Section 4.

\section{Main Results}

We first introduce some notations, which we use in the following. For the ultraspherical polynomials $P_{\lambda, n}, \lambda \neq 0$, we use the normalization $P_{\lambda, n}(1)=\left(\begin{array}{c}n+2 \lambda-1 \\ n\end{array}\right)=O\left(n^{2 \lambda-1}\right)$. We denote the zeros of $P_{\lambda, n}$ by $x_{v, n}^{(\lambda)}, v=1, \ldots, n$, and the zeros of Stieltjes polynomials $E_{\lambda, n+1}$ by $\xi_{\mu, n+1}^{(\lambda)}, \mu=1, \ldots, n+1$. We denote the zeros of $F_{\lambda, 2 n+1}:=P_{\lambda, n} E_{\lambda, n+1}$ by $y_{\nu, 2 n+1}^{(\lambda)}, v=$ $1, \ldots, 2 n+1$. All nodes are ordered by increasing magnitude. We set $\varphi(x):=\sqrt{1-x^{2}}$, and for any two sequences $\left\{b_{n}\right\}_{n}$ and $\left\{c_{n}\right\}_{n}$ of nonzero real numbers (or functions), we write $b_{n} \sim c_{n}$ if $b_{n} \leq C c_{n}$ and $c_{n} \leq C b_{n}$. We denote the space of polynomials of degree at most $n$ by $\mathscr{P}_{n}$.

For the Chebyshev polynomial $T_{n}(x)$, note that for $\lambda=0$ and $\lambda=1$

$$
\begin{gathered}
E_{0, n+1}(x)=\frac{2 n}{\pi}\left(T_{n+1}(x)-T_{n-1}(x)\right), \\
E_{1, n+1}(x)=\frac{2}{\pi} T_{n+1}(x) .
\end{gathered}
$$

In this paper, we let $P_{n}^{(0)}(x):=T_{n}(x) / n$. In these cases, the results are well known or can easily be deduced. Therefore, we will consider the cases for $0<\lambda<1$ and let $0<\lambda<1$ in the following.

Let $H_{n+1, m}[f]$ be the Hermite-Fejér interpolation polynomials of $f$ with respect to the zeros of $E_{\lambda, n+1}(x)$. Also let $\mathscr{H}_{2 n+1, m}[f]$ be the Hermite-Fejér interpolation polynomials of $f$ with respect to the zeros of $F_{\lambda, 2 n+1}(x)$. The fundamental Lagrange interpolation polynomials $l_{k, n+1}(x)$ and $l^{*}{ }_{v, 2 n+1}(x)$ with respect to $H_{n+1, m}[\cdot]$ and $\mathscr{H}_{2 n+1, m}[\cdot]$, respectively, are given by

$$
\begin{array}{r}
l_{\mu, n+1}(x)=\frac{E_{\lambda, n+1}(x)}{E_{\lambda, n+1}^{\prime}\left(\xi_{\mu, n+1}^{(\lambda)}\right)\left(x-\xi_{\mu, n+1}^{(\lambda)}\right)}, \\
\mu=1,2, \ldots, n+1, \\
l_{v, 2 n+1}^{*}(x)=\frac{F_{\lambda, 2 n+1}(x)}{F_{\lambda, 2 n+1}^{\prime}\left(y_{v, 2 n+1}^{(\lambda)}\right)\left(x-y_{v, 2 n+1}^{(\lambda)}\right)}, \\
v=1,2, \ldots, 2 n+1 .
\end{array}
$$

We let $\left\|H_{n+1, m}\right\|$ and $\left\|\mathscr{H}_{2 n+1, m}\right\|$ be the Lebesgue constants based on the zeros of $E_{\lambda, n+1}(x)$ and $F_{\lambda, 2 n+1}(x)$, respectively.
That is, the Lebesgue constants $\left\|H_{n+1, m}\right\|$ and $\left\|\mathscr{H}_{2 n+1, m}\right\|$ are defined as follows:

$$
\begin{aligned}
& \left\|H_{n+1, m}\right\| \\
& \quad:=\sup _{x \in[-1,1]} \sum_{\mu=1}^{n+1} \sum_{i=0}^{m-1}\left|e_{i, \mu} l_{\mu, n+1}^{m}(x)\left(x-\xi_{\mu, n+1}^{(\lambda)}\right)^{i}\right|, \\
& \left\|\mathscr{H}_{2 n+1, m}\right\| \\
& \quad:=\sup _{x \in[-1,1]} \sum_{v=1}^{2 n+1} \sum_{i=0}^{m-1}\left|e_{i, \nu}^{*} l_{v, 2 n+1}^{* m}(x)\left(x-y_{v, 2 n+1}^{(\lambda)}\right)^{i}\right|
\end{aligned}
$$

and for a nonnegative real function $u(x)$,

$$
\begin{aligned}
& \left\|\mathscr{H}_{2 n+1, m}\right\|_{u} \\
& \quad:=\sup _{x \in[-1,1]} u(x) \sum_{\nu=1}^{2 n+1} \sum_{i=0}^{m-1}\left|e_{i, \nu}^{*} v_{\gamma, 2 n+1}^{* m}(x)\left(x-y_{\nu, 2 n+1}^{(\lambda)}\right)^{i}\right|,
\end{aligned}
$$

where $e_{i, \mu}$ and $e_{i, v}^{*}$ are the coefficients of the higher-order Hermite-Fejér interpolation polynomials defined in (4), with respect to $H_{n+1, m}[\cdot]$ and $\mathscr{H}_{2 n+1, m}[\cdot]$, respectively.

\subsection{Uniform Convergence of Hermite-Fejér Interpolation Polynomials of Higher Order}

Theorem 1. Let $0<\lambda<1$ and $m=2,4,6, \ldots$

(a)

$$
\left\|H_{n+1, m}\right\|=O\left(n^{\max \{(1-\lambda) m-2,0\}}\right) .
$$

(b) Suppose that $(1-\lambda) m \leq 2$. Then, for a continuous function $f$ on $[-1,1]$ one has uniformly for $x \in[-1,1]$,

$$
\lim _{n \rightarrow \infty}\left|H_{n+1, m}[f](x)-f(x)\right|=0 .
$$

Theorem 2. Let $0<\lambda<1$ and $m=2,4,6, \ldots$

(a) Then one has for $0<\lambda<1 / 2$

$$
\left\|\mathscr{H}_{2 n+1, m}\right\|=O\left(n^{\max \{(1-2 \lambda) m-2,0\}}\right)
$$

and for $1 / 2 \leq \lambda<1$

$$
\left\|\mathscr{H}_{2 n+1, m}\right\|_{\varphi^{(2 \lambda-1) m}}=O(1) .
$$

(b) For a continuous function $f$ on $[-1,1]$, if $0<(1-$ $2 \lambda) m \leq 2$, then one has uniformly for $x \in[-1,1]$,

$$
\lim _{n \rightarrow \infty}\left|\mathscr{H}_{2 n+1, m}[f](x)-f(x)\right|=0
$$

and if $1 / 2 \leq \lambda<1$, then

$$
\lim _{n \rightarrow \infty}\left|\mathscr{H}_{2 n+1, m}[f](x)-f(x)\right| \varphi^{(2 \lambda-1) m}(x)=0 .
$$


2.2. Divergence of Hermite-Fejér Interpolation Polynomials of Higher Order

Theorem 3. Let $0<\lambda<1$ and $m=1,3,5, \ldots$ Then,

$$
\left\|H_{n+1, m}\right\| \sim \ln n
$$

Theorem 4. Let $0<\lambda<1$ and $m=2,4,6, \ldots$ Let $\varepsilon>0$. Suppose that

$$
\left|e_{m-1, \mu}\right| \sim n^{\lambda+m-2} \varphi^{\lambda-m}\left(\xi_{\mu, n+1}^{(\lambda)}\right) .
$$

If $(1-\lambda) m>2$, then

$$
\left\|H_{n+1, m}\right\| \sim n^{(1-\lambda) m-2} .
$$

Theorem 5. Let $0<\lambda<1$ and $m=1,3,5, \ldots$ Then,

$$
\left\|\mathscr{H}_{2 n+1, m}\right\| \sim \ln n .
$$

Theorem 6. Let $0<\lambda<1$ and $m=2,4,6, \ldots$. Let $\varepsilon>0$. Suppose that

$$
\left|e_{m-1, \nu}^{*}\right| \sim n^{\lambda+m-2} \varphi^{\lambda-m}\left(y_{v, 2 n+1}^{(\lambda)}\right) .
$$

If $(1-2 \lambda) m>2$, then

$$
\left\|\mathscr{H}_{2 n+1, m}\right\| \sim n^{\max \{(1-2 \lambda) m-2,0\}} .
$$

If the Lebesgue constant is not bounded, then we know from Helley's theorem that Hermite-Fejér interpolation does not converge for a certain continuous function on $[-1,1]$.

\section{Estimation of the Coefficients of Higher-Order Hermite-Fejér Interpolation Polynomials}

Proposition 7. Let $0<\lambda<1$.

(1) See [4, Theorem 2.1], for $n \geq 0$,

$$
\left|E_{\lambda, n+1}(x)\right| \leq C\left(n^{1-\lambda} \varphi^{1-\lambda}(x)+1\right) \quad-1 \leq x \leq 1 .
$$

Furthermore, $E_{\lambda, n+1}(1) \geq C$.

(2) See [4, Theorem 2.1] and [6, 7.33.5], for $n \geq 0$,

$$
\left|F_{\lambda, 2 n+1}(x)\right| \leq C \varphi^{1-2 \lambda}(x), \quad-1+\frac{C}{n^{2}} \leq x \leq 1-\frac{C}{n^{2}} .
$$

Proposition 8 (see [4, Lemma 5.5]). Let $0<\lambda<1$. Then, for $\mu=1,2, \ldots, n+1$,

$$
\left|E_{\lambda, n+1}^{\prime}\left(\xi_{\mu, n+1}^{(\lambda)}\right)\right| \sim n^{2-\lambda} \varphi^{-\lambda}\left(\xi_{\mu, n+1}^{(\lambda)}\right)
$$

and for $v=1,2, \ldots, 2 n+1$,

$$
\left|F_{\lambda, 2 n+1}^{\prime}\left(y_{v, 2 n+1}^{(\lambda)}\right)\right| \sim n \varphi^{-2 \lambda}\left(y_{v, 2 n+1}^{(\lambda)}\right) .
$$

Proposition 9 (see [7, Theorem 2.1]). Let $0<\lambda<1$ and $r \geq 1$ a positive integer. Then, for all $x \in\left[\xi_{1, n+1}^{(\lambda)}, \xi_{n+1, n+1}^{(\lambda)}\right]$,

$$
\left|E_{\lambda, n+1}^{(r)}(x)\right| \leq C n^{r+1-\lambda} \varphi^{1-r-\lambda}(x) .
$$

Moreover, one has for $x \in\left[-1, \xi_{1, n+1}^{(\lambda)}\right] \cup\left[\xi_{n+1, n+1}^{(\lambda)}, 1\right]$,

$$
\left|E_{\lambda, n+1}^{(r)}(x)\right| \sim n^{2 r} .
$$

Proposition 10 (see [7, Theorem 2.2]). Let $0<\lambda<1$ and $r \geq 1$ a positive integer. Then, for all $x \in\left[\xi_{1, n+1}^{(\lambda)}, \xi_{n+1, n+1}^{(\lambda)}\right]$,

$$
\left|F_{\lambda, 2 n+1}^{(r)}(x)\right| \leq \operatorname{Cn}^{r} \varphi^{1-2 \lambda-r}(x) .
$$

Moreover, one has for $x \in\left[-1, \xi_{1, n+1}^{(\lambda)}\right] \cup\left[\xi_{n+1, n+1}^{(\lambda)}, 1\right]$,

$$
\left|F_{\lambda, 2 n+1}{ }^{(r)}(x)\right| \sim n^{2 \lambda+2 r-1} .
$$

Proposition 11 (see [7, Theorems 2.3, 2.4]). Let $0<\lambda<1$ and $r \geq 2$ an even integer.

(a) For $1 \leq \mu \leq n+1$,

$$
\left|E_{\lambda, n+1}^{(r)}\left(\xi_{\mu, n+1}^{(\lambda)}\right)\right| \leq \operatorname{Cn}^{r} \varphi^{-r}\left(\xi_{\mu, n+1}^{(\lambda)}\right) .
$$

(b) For $1 \leq v \leq 2 n+1$,

$$
\left|F_{\lambda, 2 n+1}^{(r)}\left(y_{v, 2 n+1}^{(\lambda)}\right)\right| \leq C n^{r-1+\lambda} \varphi^{-r-\lambda}\left(y_{v, 2 n+1}^{(\lambda)}\right) .
$$

Proposition 12 (see [7, Lemma 4.9]). For $1 \leq v \leq 2 n+1$, one has

$$
\left|E_{\lambda, n+1}\left(x_{\nu, n}^{(\lambda)}\right)\right| \sim n^{1-\lambda} \varphi^{1-\lambda}\left(x_{\nu, n}^{(\lambda)}\right) .
$$

Proposition 13 (see [7, Theorems 2.6, 2.7]). Let $0<\lambda<1$ and $0<\varepsilon<1$.

(a) For $\left|\xi_{\mu, n+1}^{(\lambda)}\right| \leq 1-\varepsilon$ and a positive integer $\ell \geq 0$, one has

$$
\begin{aligned}
E_{\lambda, n+1}^{(2 \ell+1)}\left(\xi_{\mu, n+1}^{(\lambda)}\right)= & (-1)^{\ell}(n+1)^{2 \ell} \varphi^{-2 \ell} \\
& \times\left(\xi_{\mu, n+1}^{(\lambda)}\right) E_{\lambda, n+1}^{\prime}\left(\xi_{\mu, n+1}^{(\lambda)}\right)+O\left(n^{2 \ell+1}\right) .
\end{aligned}
$$

(b) For $\left|y_{v, 2 n+1}^{(\lambda)}\right| \leq 1-\varepsilon$ and a positive integer $\ell \geq 0$, one has

$$
\begin{aligned}
F_{\lambda, 2 n+1}^{(2 \ell+1)}\left(y_{v, 2 n+1}^{(\lambda)}\right)= & c_{\ell}(-1)^{\ell}(n+1)^{2 \ell} \varphi^{-2 \ell}\left(y_{v, 2 n+1}^{(\lambda)}\right) \\
& \times F_{\lambda, 2 n+1}^{\prime}\left(y_{v, 2 n+1}^{(\lambda)}\right)+O\left(n^{\lambda+2 \ell}\right),
\end{aligned}
$$$$
\text { where } c_{\ell}=2^{\ell-1}+4^{\ell} / 2 \text {. }
$$

Theorem 14. Let $0<\lambda<1, r \geq 0$ and $n, m \geq 1$.

(a) Uniformly for $1 \leq \mu \leq n+1$

$$
\left|\left[l_{\mu, n+1}^{m}\right]^{(r)}\left(\xi_{\mu, n+1}^{(\lambda)}\right)\right| \leq \operatorname{Cn}^{r} \varphi^{-r}\left(\xi_{\mu, n+1}^{(\lambda)}\right)
$$

and if $r$ is odd,

$$
\left|\left[l_{\mu, n+1}^{m}\right]^{(r)}\left(\xi_{\mu, n+1}^{(\lambda)}\right)\right| \leq C n^{\lambda+r-1} \varphi^{-r-1+\lambda}\left(\xi_{\mu, n+1}^{(\lambda)}\right) .
$$


Journal of Applied Mathematics

5

(b) Uniformly for $0 \leq s \leq m-1$,

$$
\left|e_{s, \mu}\right| \leq \operatorname{Cn}^{s} \varphi^{-s}\left(\xi_{\mu, n+1}^{(\lambda)}\right)
$$

and ifs is odd,

$$
\left|e_{s, \mu}\right| \leq C n^{\lambda+s-1} \varphi^{-s-1+\lambda}\left(\xi_{\mu, n+1}^{(\lambda)}\right) .
$$

Theorem 15. Let $0<\lambda<1, r \geq 0$ and $n, m \geq 1$.

(a) Uniformly for $1 \leq v \leq 2 n+1$,

$$
\left|\left[l_{v, 2 n+1}^{* m}\right]^{(r)}\left(y_{v, 2 n+1}^{\lambda}\right)\right| \leq \operatorname{Cn}^{r} \varphi^{-r}\left(y_{v, 2 n+1}^{\lambda}\right)
$$

and if $r$ is odd, one has

$$
\left|\left[l_{v, 2 n+1}^{* m}\right]^{(r)}\left(y_{v, 2 n+1}^{\lambda}\right)\right| \leq C n^{\lambda+r-1} \varphi^{-r-1+\lambda}\left(y_{v, 2 n+1}^{\lambda}\right) .
$$

(b) Uniformly for $0 \leq s \leq m-1$,

$$
\left|e_{s, v}^{*}\right| \leq C n^{s} \varphi^{-s}\left(y_{v, 2 n+1}^{\lambda}\right)
$$

and if $s$ is odd,

$$
\left|e_{s, \nu}^{*}\right| \leq C n^{\lambda+s-1} \varphi^{-s-1+\lambda}\left(y_{v, 2 n+1}^{\lambda}\right) .
$$

Theorem 16. Let $0<\lambda<1$ and $0<\varepsilon<1$. Suppose that $\left|\xi_{\mu, n+1}^{(\lambda)}\right| \leq 1-\varepsilon$. Then, there exists a constant $\phi_{\ell}(m)>0$ depending only on $m$ and $\ell$ such that

$$
\begin{aligned}
\left(l_{\mu, n+1}^{m}\right)^{(2 \ell)}\left(\xi_{\mu, n+1}^{(\lambda)}\right)= & (-1)^{\ell} \phi_{\ell}(m)(n+1)^{2 \ell} \\
& \times \varphi^{-2 \ell}\left(\xi_{\mu, n+1}^{(\lambda)}\right)\left(1+O\left(n^{\lambda-1}\right)\right) .
\end{aligned}
$$

Theorem 17. Let $0<\lambda<1$ and $0<\varepsilon<1$. Suppose that $\left|\xi_{\mu, n+1}^{(\lambda)}\right| \leq 1-\varepsilon$. Then, there exists a constant $\Psi_{\ell}(-m)$ with $\Psi_{\ell}(-m)>0$ depending only on $m$ and $\ell$ such that

$$
\begin{aligned}
e_{2 \ell, \mu}= & (-1)^{\ell} \frac{1}{(2 \ell) !} \Psi_{\ell}(-m)(n+1)^{2 \ell} \varphi^{-2 \ell}\left(\xi_{\mu, n+1}^{(\lambda)}\right) \\
& \times\left(1+O\left(n^{\lambda-1}\right)\right) .
\end{aligned}
$$

Theorem 18. Let $0<\lambda<1$ and $0<\varepsilon<1$. Suppose that $\left|y_{\nu, 2 n+1}^{(\lambda)}\right| \leq 1-\varepsilon$. Then, there exists a constant $\alpha_{\ell}(m)>0$ depending only on $m$ and $\ell$ such that

$$
\begin{aligned}
\left(l_{v, 2 n+1}^{* m}\right)^{(2 \ell)}\left(y_{v, 2 n+1}^{(\lambda)}\right)= & (-1)^{\ell} \alpha_{\ell}(m)(n+1)^{2 \ell} \\
& \times \varphi^{-2 \ell}\left(y_{v, 2 n+1}^{(\lambda)}\right)\left(1+O\left(n^{\lambda-1}\right)\right) .
\end{aligned}
$$

Theorem 19. Let $0<\lambda<1$ and $0<\varepsilon<1$. Suppose that $\left|y_{v, 2 n+1}^{(\lambda)}\right| \leq 1-\varepsilon$. Then, there exists a constant $\Phi_{\ell}(-m)$ with $\Phi_{\ell}(-m)>0$ depending only on $m$ and $\ell$ such that

$$
\begin{aligned}
e_{2 \ell, v}^{*}= & (-1)^{\ell} \frac{1}{(2 \ell) !} \Phi_{\ell}(-m)(n+1)^{2 \ell} \varphi^{-2 \ell}\left(y_{\nu, 2 n+1}^{(\lambda)}\right) \\
& \times\left(1+O\left(n^{\lambda-1}\right)\right) .
\end{aligned}
$$

Proof of Theorem 14. We prove by induction on $m$. Since

$$
\begin{aligned}
& {\left[E_{\lambda, n+1}^{\prime}\left(\xi_{\mu, n+1}^{(\lambda)}\right)\left(x-\xi_{\mu, n+1}^{(\lambda)}\right) l_{\mu, n+1}(x)\right]_{x=\xi_{\mu, n+1}^{(\lambda)}}^{(r+1)}} \\
& =E_{\lambda, n+1}^{(r+1)}\left(\xi_{\mu, n+1}^{(\lambda)}\right)
\end{aligned}
$$

we know that

$$
l_{\mu, n+1}^{(r)}\left(\xi_{\mu, n+1}^{(\lambda)}\right)=\frac{E_{\lambda, n+1}^{(r+1)}\left(\xi_{\mu, n+1}^{(\lambda)}\right)}{(r+1) E_{\lambda, n+1}^{\prime}\left(\xi_{\mu, n+1}^{(\lambda)}\right)} .
$$

So, it holds for $m=1$ by (34) and (36). Now, assume that it holds for $1,2, \ldots, m-1$. Then, using Leibnitz's rule for differentiation, we obtain

$$
\begin{aligned}
\left|\left[l_{\mu, n+1}^{m}\right]^{(r)}\left(\xi_{\mu, n+1}^{(\lambda)}\right)\right| \leq & C \sum_{i=0}^{r}\left(\begin{array}{c}
r \\
i
\end{array}\right)\left|l_{\mu, n+1}^{(i)}\left(\xi_{\mu, n+1}^{(\lambda)}\right)\right| \\
& \times\left|\left[l_{\mu, n+1}^{m-1}\right]^{(r-i)}\left(\xi_{\mu, n+1}^{(\lambda)}\right)\right| \\
\leq & \operatorname{Cn}^{r} \varphi^{-r}\left(\xi_{\mu, n+1}^{(\lambda)}\right) .
\end{aligned}
$$

Suppose that $r$ is odd. Then,

$$
\begin{aligned}
\left|\left[l_{\mu, n+1}^{m}\right]^{(r)}\left(\xi_{\mu, n+1}^{(\lambda)}\right)\right| \leq & C \sum_{i=0}^{r}\left(\begin{array}{l}
r \\
i
\end{array}\right)\left|l_{\mu, n+1}^{(i)}\left(\xi_{\mu, n+1}^{(\lambda)}\right)\right| \\
& \times\left|\left[l_{\mu, n+1}^{m-1}\right]^{(r-i)}\left(\xi_{\mu, n+1}^{(\lambda)}\right)\right| \\
: & \left(\sum_{i=\text { even }}+\sum_{i=\text { odd }}\right) .
\end{aligned}
$$

Since $r-i$ is odd for an even $i$ and $r-i$ is even for an odd $i$, we have by the mathematical induction on $m,(34),(36),(40)$, and (58),

$$
\begin{gathered}
\sum_{i=\text { even or odd }}\left|l_{\mu, n+1}^{(i)}\left(\xi_{\mu, n+1}^{(\lambda)}\right)\right|\left|\left[l_{\mu, n+1}^{m-1}\right]^{(r-i)}\left(\xi_{\mu, n+1}^{(\lambda)}\right)\right| \\
\leq C n^{\lambda+r-1} \varphi^{-r+\lambda-1}\left(\xi_{\mu, n+1}^{(\lambda)}\right) .
\end{gathered}
$$

These complete the proofs of (45) and (46). To prove (47) and (48), we proceed by induction on $s$. Firstly, for $s=0$, (47) is trivial since $e_{0, \mu}=1$. For $s \geq 1$, we have by $[8,(3.3)]$ and $[8$, (3.4)]

$$
0=h_{\mu}^{(s)}\left(\xi_{\mu, n+1}^{(\lambda)}\right)=\sum_{i=0}^{s} e_{i, \mu}\left(\begin{array}{l}
s \\
i
\end{array}\right) i !\left[l_{\mu, n+1}^{m}\right]^{(s-i)}\left(\xi_{\mu, n+1}^{(\lambda)}\right),
$$

so that

$$
e_{s, \mu}=-\frac{1}{s !} \sum_{i=0}^{s-1} e_{i, \mu}\left(\begin{array}{l}
s \\
i
\end{array}\right) i !\left[l_{\mu, n+1}^{m}\right]^{(s-i)}\left(\xi_{\mu, n+1}^{(\lambda)}\right) .
$$

Thus, if we assume that (47) holds for $s=0,1, \ldots, t-1, t \geq 1$, then by (45), we have

$$
\left|e_{t, \mu}\right| \leq C \sum_{i=0}^{t-1}\left|e_{i, \mu}\right|\left[l_{\mu, n+1}^{m}\right]^{(t-i)}\left(\xi_{\mu, n+1}^{(\lambda)}\right) \leq C \frac{n^{t}}{\varphi^{t}\left(\xi_{\mu, n+1}^{(\lambda)}\right)} .
$$


Suppose that $t$ is odd. Then,

$$
\left|e_{t, \mu}\right| \leq C\left(\sum_{i=\text { even }}^{t-1}+\sum_{i=\text { odd }}^{t-1}\right)\left|e_{i, \mu}\right|\left[l_{\mu, n+1}^{m}\right]^{(t-i)}\left(\xi_{\mu, n+1}^{(\lambda)}\right) .
$$

Since $t-i$ is odd for an even $i$ and $t-i$ is even for an odd $i$, we have by the mathematical induction, (45), (46), and (47)

$$
\begin{array}{r}
\sum_{i=\text { even or odd }}^{t-1}\left|e_{i, \mu}\right|\left[l_{\mu, n+1}^{m}\right]^{(t-i)}\left(\xi_{\mu, n+1}^{(\lambda)}\right) \\
\leq C n^{\lambda+t-1} \varphi^{\lambda-t-1}\left(\xi_{\mu, n+1}^{(\lambda)}\right) .
\end{array}
$$

These complete the proofs of (47) and (48).

Proof of Theorem 15. Using (35), (38), and (41), this is proved by the same method as the proof of Theorem 14 .

For $j=0,1, \ldots$ define $\phi_{j}(1):=(2 j+1)^{-1}$ and for $k \geq 2$

$$
\phi_{j}(k):=\sum_{r=0}^{j} \frac{1}{2 j-2 r+1}\left(\begin{array}{l}
2 j \\
2 r
\end{array}\right) \phi_{r}(k-1) .
$$

We rewrite the relation (67) in the form for $v=1,2,3 \ldots$,

$$
\phi_{0}(\nu):=1
$$

and for $j=1,2,3, \ldots, v=2,3,4, \ldots$,

$$
\phi_{j}(\nu)-\phi_{j}(\nu-1)=\frac{1}{2 j+1} \sum_{r=0}^{j-1}\left(\begin{array}{c}
2 j+1 \\
2 r
\end{array}\right) \phi_{r}(\nu-1) .
$$

Now, for every $j$, we will introduce an auxiliary polynomial determined by $\left\{\Psi_{j}(y)\right\}_{j=1}^{\infty}$ as the following lemma.

Lemma 20 (see [9, Lemma 11]).

(i) For $j=0,1,2, \ldots$, there exists a unique polynomial $\Psi_{j}(y)$ of degree $j$ such that

$$
\Psi_{j}(\nu)=\phi_{j}(\nu), \quad \nu=1,2,3, \ldots
$$

(ii) $\Psi_{0}(y)=1$ and $\Psi_{j}(0)=0, j=1,2, \ldots$

Lemma 21 (see [9, Lemma 13]). If $y<0$, then for $j=$ $0,1,2, \ldots$,

$$
(-1)^{j} \Psi_{j}(y)>0
$$

Lemma 22 (see [9, Proof of Lemma 14]). For positive integers $s$ and $m$,

$$
\sum_{r=0}^{s}\left(\begin{array}{l}
2 s \\
2 r
\end{array}\right) \Psi_{r}(-m) \phi_{s-r}(m)=0 .
$$

Proof of Theorem 16. Similarly to Theorem 14, we use mathematical induction with respect to $m$. From (58), (43), and (34), we know that

$$
\begin{aligned}
& l_{\mu, n+1}^{(2 \ell)}\left(\xi_{\mu, n+1}\right)=(-1)^{\ell} \phi_{\ell}(1)(n+1)^{2 \ell} \varphi^{-2 \ell}\left(\xi_{\mu, n+1}^{(\lambda)}\right) \\
& \times\left(1+O\left(n^{\lambda-1}\right)\right) \\
&\left|l_{\mu, n+1}^{(2 \ell-1)}\left(\xi_{\mu, n+1}\right)\right| \leq O\left(n^{2 \ell-2+\lambda}\right) .
\end{aligned}
$$

Then, from the following relations:

$$
\begin{aligned}
& \left(l_{\mu, n+1}^{m}\right)^{(2 \ell)}\left(\xi_{\mu, n+1}\right) \\
& =\sum_{0 \leq 2 r \leq 2 \ell}\left(\begin{array}{l}
2 \ell \\
2 r
\end{array}\right)\left(l_{\mu, n+1}^{m-1}\right)^{(2 r)}\left(\xi_{\mu, n+1}\right) l_{\mu, n+1}^{(2 \ell-2 r)}\left(\xi_{\mu, n+1}\right) \\
& +\sum_{1 \leq 2 r-1 \leq 2 \ell}\left(\begin{array}{c}
2 \ell \\
2 r-1
\end{array}\right)\left(l_{\mu, n+1}^{m-1}\right)^{(2 r-1)} \\
& \times\left(\xi_{\mu, n+1}\right) l_{\mu, n+1}^{(2 \ell-2 r+1)}\left(\xi_{\mu, n+1}\right) \\
& =(-1)^{\ell} \phi_{\ell}(m)(n+1)^{2 \ell} \varphi^{-2 \ell}\left(\xi_{\mu, n+1}^{(\lambda)}\right)\left(1+O\left(n^{\lambda-1}\right)\right) \text {, } \\
& \left(l_{\mu, n+1}^{m}\right)^{(2 \ell-1)}\left(\xi_{\mu, n+1}\right) \\
& =\sum_{0 \leq 2 r \leq 2 \ell-1}\left(\begin{array}{c}
2 \ell-1 \\
2 r
\end{array}\right)\left(l_{\mu, n+1}^{m-1}\right)^{(2 r)}\left(\xi_{\mu, n+1}\right) l_{\mu, n+1}^{(2 \ell-2 r-1)}\left(\xi_{\mu, n+1}\right) \\
& +\sum_{1 \leq 2 r-1 \leq 2 \ell-1}\left(\begin{array}{c}
2 \ell-1 \\
2 r-1
\end{array}\right)\left(l_{\mu, n+1}^{m-1}\right)^{(2 r-1)} \\
& \times\left(\xi_{\mu, n+1}\right) l_{\mu, n+1}^{(2 \ell-2 r)}\left(\xi_{\mu, n+1}\right) \\
& =O\left(n^{2 \ell-2+\lambda}\right) \text {, }
\end{aligned}
$$

we have the results by induction with respect to $m$.

Proof of Theorem 17. We prove (54) by induction on $s$. Since $e_{0, \mu}=1$ and $\Psi_{0}(y)=1,(54)$ holds for $s=0$. From (63), we write $e_{2 s, \mu}$ in the form of

$$
\begin{aligned}
e_{2 s, \mu}= & -\sum_{r=0}^{s-1} \frac{1}{(2 s-2 r) !} e_{2 r, \mu}\left(l_{\mu, n+1}^{m}\right)^{(2 s-2 r)}\left(\xi_{\mu, n+1}^{(\lambda)}\right) \\
& -\sum_{r=1}^{s} \frac{1}{(2 s-2 r+1) !} e_{2 r-1, \mu}\left(l_{\mu, n+1}^{m}\right)^{(2 s-2 r+1)}\left(\xi_{\mu, n+1}^{(\lambda)}\right) \\
= & I+I I .
\end{aligned}
$$

Then, by (46) and (48), $|I I|$ is $O\left(n^{2 s+2 \lambda-2}\right)$. For $0 \leq r \leq s-1$, we suppose (54). Then, since we know from (53)

$$
\begin{aligned}
\left(l_{\mu, n+1}^{m}\right)^{(2 s-2 r)}\left(\xi_{\mu, n+1}^{(\lambda)}\right)= & (-1)^{s-r} \phi_{s-r}(m)(n+1)^{2(s-r)} \\
& \times \varphi^{-2(s-r)}\left(\xi_{\mu, n+1}^{(\lambda)}\right)\left(1+O\left(n^{\lambda-1}\right)\right), \\
e_{2 r, \mu}= & (-1)^{r} \frac{1}{(2 r) !} \Psi_{r}(-m)(n+1)^{2 r} \\
& \times \varphi^{-2 r}\left(\xi_{\mu, n+1}^{(\lambda)}\right)\left(1+O\left(n^{\lambda-1}\right)\right),
\end{aligned}
$$


we have for $I$

$$
\begin{aligned}
\sum_{r=0}^{s-1}= & \frac{(-1)^{s+1}}{(2 s) !}(n+1)^{2 s} \varphi^{-2 s}\left(\xi_{\mu, n+1}^{(\lambda)}\right) \\
& \times \sum_{r=0}^{s-1}\left(\begin{array}{l}
2 s \\
2 r
\end{array}\right) \Psi_{r}(-m) \phi_{s-r}(m)\left(1+O\left(n^{\lambda-1}\right)\right) .
\end{aligned}
$$

Then, using Lemma 22 and $\phi_{0}(m)=1$, we have the following form:

$$
\begin{aligned}
e_{2 s, \mu}= & \frac{(-1)^{s}}{(2 s) !} \Psi_{s}(-m)(n+1)^{2 s} \varphi^{-2 s}\left(\xi_{\mu, n+1}^{(\lambda)}\right) \\
& \times\left(1+O\left(n^{\lambda-1}\right)\right) .
\end{aligned}
$$

Therefore, we proved the result.

Proof of Theorems 18 and 19. These theorems are proved by the same method as the above theorems.

\section{The Proofs of Theorems}

4.1. Proof of Theorems 1 and 2. From now on, we assume that $0<\lambda<1$. We first state some known results for the Stieltjes polynomials. Let $x_{\eta, n}^{(\lambda)}:=\cos \phi_{\eta, n}^{(\lambda)}, \eta=1, \ldots, n$, $\xi_{\mu, n+1}^{(\lambda)}:=\cos \theta_{\mu, n+1}^{(\lambda)}, \mu=1, \ldots, n+1$, and $y_{\nu, 2 n+1}^{(\lambda)}:=\cos \psi_{\nu, 2 n+1}^{(\lambda)}$, $\nu=1,2, \ldots, 2 n+1$. Ehrich and Mastroianni [4] proved that for $\mu=0,1, \ldots, n+1$ and $\nu=0,1, \ldots, 2 n+1$

$$
\begin{aligned}
\left|\phi_{\eta, n}^{(\lambda)}-\phi_{\eta+1, n}^{(\lambda)}\right| & \sim\left|\theta_{\mu, n+1}^{(\lambda)}-\theta_{\mu+1, n+1}^{(\lambda)}\right| \\
& \sim\left|\psi_{\nu, 2 n+1}^{(\lambda)}-\psi_{\nu+1,2 n+1}^{(\lambda)}\right| \sim n^{-1},
\end{aligned}
$$

where $\psi_{0,2 n+1}^{(\lambda)}:=\theta_{0, n+1}^{(\lambda)}:=\phi_{0, n}^{(\lambda)}=\pi$ and $\psi_{2 n+2,2 n+1}^{(\lambda)}:=$ $\theta_{n+2, n+1}^{(\lambda)}:=\phi_{n+1, n}^{(\lambda)}:=0$. It implies that for $\mu=0,1, \ldots, n+2$ and $\nu=0,1, \ldots, 2 n+2$,

$$
\begin{aligned}
\xi_{\mu+1, n+1}^{(\lambda)}-\xi_{\mu, n+1}^{(\lambda)} & \sim \frac{1}{n} \varphi\left(\xi_{\mu, n+1}^{(\lambda)}\right) ; \\
y_{\nu+1,2 n+1}^{(\lambda)}-y_{\nu, 2 n+1}^{(\lambda)} & \sim \frac{1}{n} \varphi\left(y_{v, 2 n+1}^{(\lambda)}\right), \\
\varphi\left(\xi_{\mu+1, n+1}^{(\lambda)}\right) & \sim \varphi\left(\xi_{\mu, n+1}^{(\lambda)}\right) ; \\
\varphi\left(y_{\nu+1,2 n+1}^{(\lambda)}\right) & \sim \varphi\left(y_{v, 2 n+1}^{(\lambda)}\right),
\end{aligned}
$$

where $y_{0,2 n+1}^{(\lambda)}:=\xi_{0, n+1}^{(\lambda)}:=-1$ and $y_{2 n+2,2 n+1}^{(\lambda)}:=\xi_{n+2, n+1}^{(\lambda)}:=1$.

Lemma 23. Let $k$ be a positive integer and $0<x<1-C / n^{2}$. Then,

(a) for $\alpha>0$,

$$
\begin{aligned}
& \int_{\substack{0 \leq t \leq 1-C / n^{2},|x-t|>\varphi(x) / n}} \frac{\varphi^{-\alpha}(t) \varphi^{k-1}(t)}{|x-t|^{k}} d t \\
& \leq C \varphi^{-\alpha}(x) n^{k-1} O\left(n^{\max \{\alpha-2 k, 0\}}\right) \\
& \quad \times \begin{cases}1, & k \geqslant 2 ; \\
\ln n, & k=1 ;\end{cases}
\end{aligned}
$$

(b) for $\alpha_{1}>0$ and $\varepsilon_{1}>0$,

$$
\begin{aligned}
& \int_{\substack{0 \leq t \leq 1-C / n^{2},|x-t|>\varphi(x) / n}} \frac{\varphi^{-\alpha_{1}-\varepsilon_{1}}(t) \varphi^{k-1}(t)}{|x-t|^{k}} d t \\
& \quad \leq C \varphi^{-\alpha_{1}}(x) n^{k-1+\varepsilon_{1}} O\left(n^{\max \left\{\alpha_{1}-2 k, 0\right\}}\right)
\end{aligned}
$$

(c) for $\alpha>0$,

$$
\int_{\substack{0 \leq t \leq 1-C / n^{2},|x-t|>\varphi(x) / n}} \frac{\varphi^{\alpha}(t) \varphi^{k-1}(t)}{|x-t|^{k}} d t \leq C n^{k-1} \begin{cases}1, & k \geqslant 2 ; \\ \ln n, & k=1 ;\end{cases}
$$

(d) for $\alpha_{1}>0$ and $\varepsilon_{1}>0$,

$$
\int_{\substack{0 \leq t \leq 1-C / n^{2},|x-t|>\varphi(x) / n}} \frac{\varphi^{\alpha_{1}-\varepsilon_{1}}(t) \varphi^{k-1}(t)}{|x-t|^{k}} d t \leq C n^{k-1+\varepsilon_{1}} .
$$

Proof. (a)

$$
\begin{aligned}
\int_{\substack{0 \leq t \leq 1-C / n^{2},|x-t|>\varphi(x) / n}} \frac{\varphi^{-\alpha+k-1}(t)}{|x-t|^{k}} d t \\
\quad \int_{0}^{x_{*}}+\int_{x^{*}}^{\left(1+x^{*}\right) / 2}+\int_{\left(1+x^{*}\right) / 2}^{1-C / n^{2}} \frac{\varphi^{-\alpha+k-1}(t)}{|x-t|^{k}} d t \\
=: I_{1}+I_{2}+I_{3},
\end{aligned}
$$

where $x_{*}:=x-\varphi(x) / n$ and $x^{*}:=x+\varphi(x) / n$.

$I_{1}$ : Suppose that $0 \leq t \leq x_{*}$. Then, since $\varphi^{-\alpha}(t) \leq \varphi^{-\alpha}(x)$, we have

$$
\int_{0}^{x_{*}} \frac{\varphi^{-\alpha+k-1}(t)}{|x-t|^{k}} d t \leq C \varphi^{-\alpha}(x) \int_{0}^{x_{*}} \frac{\varphi^{k-1}(t)}{|x-t|^{k}} d t .
$$

Since we know for $0 \leq t \leq x_{*}$,

$$
\varphi(t) \sim(x-t)^{1 / 2}+\varphi(x)
$$

we have

$$
\begin{aligned}
\int_{0}^{x_{*}} & \frac{\varphi^{k-1}(t)}{|x-t|^{k}} d t \\
\sim & \int_{0}^{x_{*}} \frac{1}{|x-t|^{(k-1) / 2}|x-t|} d t+\varphi^{k-1}(x) \\
& \times \int_{0}^{x_{*}} \frac{1}{|x-t|^{k-1}|x-t|} d t \\
\leq & C \begin{cases}\left|x-x_{*}\right|^{-(k-1) / 2}+\varphi^{k-1}(x)\left|x-x_{*}\right|^{-k+1}, & k \geq 2 ; \\
\ln n, & k=1\end{cases} \\
\leq & C \begin{cases}n^{k-1}, & k \geq 2 ; \\
\ln n, & k=1 .\end{cases}
\end{aligned}
$$


Therefore, we have for $I_{1}$

$$
I_{1} \leq C \varphi^{-\alpha}(x) \begin{cases}n^{k-1}, & k \geq 2 \\ \ln n, & k=1\end{cases}
$$

$I_{2}:$ Since $\varphi(t) \sim \varphi(x)$ for $x^{*} \leq t \leq\left(1+x^{*}\right) / 2$,

$$
\begin{aligned}
\int_{x^{*}}^{\left(1+x^{*}\right) / 2} & \frac{\varphi^{-\alpha+k-1}(t)}{|x-t|^{k}} d t \\
& \sim \varphi^{-\alpha+k-1}(x) \int_{x^{*}}^{\left(1+x^{*}\right) / 2} \frac{1}{|x-t|^{k}} d t \\
& \leq C \varphi^{-\alpha}(x) \begin{cases}n^{k-1}, & k \geq 2 ; \\
\ln n, & k=1 .\end{cases}
\end{aligned}
$$

Therefore, we have

$$
I_{2} \leq C \varphi^{-\alpha}(x) \begin{cases}n^{k-1}, & k \geq 2 \\ \ln n, & k=1\end{cases}
$$

$I_{3}$ : Since $|x-t| \geq C|1-t|$ for $\left(1+x^{*}\right) / 2<t<1-C / n^{2}$, we have for $I_{3}$

$$
\begin{aligned}
& \int_{\left(1+x^{*}\right) / 2}^{1-C / n^{2}} \frac{\varphi^{-\alpha+k-1}(t)}{|x-t|^{k}} d t \\
& \quad=\int_{\left(1+x^{*}\right) / 2}^{1-C / n^{2}} \frac{\varphi^{-\alpha+k-1}(t)}{|x-t|^{k-\alpha / 2}|x-t|^{\alpha / 2}} d t \\
& \leq C \frac{1}{\left|\left(1+x^{*}\right) / 2-x\right|^{\alpha / 2}} \\
& \quad \times \int_{\left(1+x^{*}\right) / 2}^{1-C / n^{2}} \frac{1}{(1-t)^{(k+1) / 2}} \frac{\varphi^{-\alpha+2 k}(t)}{|x-t|^{-\alpha / 2+k}} d t .
\end{aligned}
$$

Since for $\left(1+x^{*}\right) / 2<t<1-C / n^{2}$,

$$
\begin{aligned}
& \frac{\varphi^{-\alpha+2 k}(t)}{|x-t|^{-\alpha / 2+k}} \\
& \quad \leq C \begin{cases}1, & \text { if }-\alpha+2 k \geq 0 ; \\
n^{\alpha-2 k}, & \text { if }-\alpha+2 k<0\end{cases} \\
& \quad=O\left(n^{\max \{\alpha-2 k, 0\}}\right),
\end{aligned}
$$

with $\left|\left(1+x^{*}\right) / 2-x\right| \sim 1-x \sim \varphi^{2}(x)$, we have

$$
\begin{aligned}
I_{3}= & \int_{\left(1+x^{*}\right) / 2}^{1-C / n^{2}} \frac{\varphi^{-\alpha+k-1}(t)}{|x-t|^{k}} d t \\
\leq & O\left(n^{\max \{\alpha-2 k, 0\}}\right) \varphi^{-\alpha}(x) \\
& \times \int_{\left(1+x^{*}\right) / 2}^{1-C / n^{2}} \frac{1}{(1-t)^{(k+1) / 2}} d t \\
\sim & n^{k-1} \varphi^{-\alpha}(x) O\left(n^{\max \{\alpha-2 k, 0\}}\right) \begin{cases}1, & k \geqslant 2 ; \\
\ln n, & k=1 .\end{cases}
\end{aligned}
$$

Therefore, we have the result (a). (b) Similarly to (a), we let

$$
\begin{aligned}
& \int_{\substack{0 \leq t \leq 1-C / n^{2},|x-t|>\varphi(x) / n}} \frac{\varphi^{-\alpha_{1}-\varepsilon_{1}+k-1}(t)}{|x-t|^{k}} d t \\
& \sim \int_{0}^{x_{*}}+\int_{x^{*}}^{\left(1+x^{*}\right) / 2}+\int_{\left(1+x^{*}\right) / 2}^{1-C / n^{2}} \frac{\varphi^{-\alpha_{1}+k-1-\epsilon_{1}}(t)}{|x-t|^{k}} d t \\
& \quad=: I_{1}+I_{2}+I_{3} .
\end{aligned}
$$

Then, for $I_{1}$, we have using (87)

$$
\begin{aligned}
& \int_{0}^{x_{*}} \frac{\varphi^{-\alpha_{1}+k-1-\epsilon_{1}}(t)}{|x-t|^{k}} d t \\
& \leq C \varphi^{-\alpha_{1}}(x) \int_{0}^{x_{*}}\left(\frac{\varphi^{-\epsilon_{1}}(t)}{|x-t|^{(k-1) / 2}|x-t|}\right. \\
& \left.+\frac{\varphi^{k-1}(x) \varphi^{-\epsilon_{1}}(t)}{|x-t|^{k-1}|x-t|}\right) d t \\
& \leq C \varphi^{-\alpha_{1}}(x) n^{k-1} \int_{0}^{x_{*}} \frac{\varphi^{-\epsilon_{1}}(t)}{x-t} d t, \\
& \int_{0}^{x_{*}} \frac{\varphi^{-\epsilon_{1}}(t)}{x-t} d t \leq C \int_{0}^{x_{*}} \frac{1}{(x-t)^{\epsilon_{1} / 2+1}} d t \\
& \leq C\left(\frac{n}{\varphi(x)}\right)^{\epsilon_{1} / 2} \leq C n^{\varepsilon_{1}}
\end{aligned}
$$

by the use of $\varphi(t) \geq C|x-t|^{1 / 2}$. Therefore, we have for $I_{1}$

$$
I_{1} \leq C \varphi^{-\alpha_{1}}(x) n^{k-1+\varepsilon_{1}}
$$

$I_{2}$ : Since $1-t \geq C(t-x)$ and $\varphi(t) \sim \varphi(x)$ for $x^{*} \leq t \leq$ $\left(1+x^{*}\right) / 2$,

$$
\begin{aligned}
& \int_{x^{*}}^{\left(1+x^{*}\right) / 2} \frac{\varphi^{-\alpha_{1}+k-1-\epsilon_{1}}(t)}{|x-t|^{k}} d t \\
& \quad \sim \varphi^{-\alpha_{1}}(x) n^{k-1} \int_{x^{*}}^{\left(1+x^{*}\right) / 2} \frac{\varphi^{-\epsilon_{1}}(t)}{(t-x)} d t \\
& \quad \leq C \varphi^{-\alpha_{1}}(x) n^{k-1} \int_{x^{*}}^{\left(1+x^{*}\right) / 2} \frac{1}{(t-x)^{\epsilon_{1} / 2+1}} d t \\
& \quad \leq C \varphi^{-\alpha_{1}}(x) n^{k-1+\varepsilon_{1}} .
\end{aligned}
$$

Therefore,

$$
I_{2} \leq C \varphi^{-\alpha_{1}}(x) n^{k-1+\varepsilon_{1}}
$$


$I_{3}:$ Since $|x-t| \geq C\left|\left(1+x^{*}\right) / 2-x\right|$ for $\left(1+x^{*}\right) / 2<t<$ $1-C / n^{2}$, we have using (93)

$$
\begin{aligned}
& \frac{\varphi^{-\alpha_{1}+k-1-\epsilon_{1}}(t)}{|x-t|^{k}} \\
& \quad=\frac{\varphi^{-\alpha_{1}+k-1-\epsilon_{1}}(t)}{|x-t|^{k-\alpha_{1} / 2}|x-t|^{\alpha_{1} / 2}} \\
& \quad \leq C \frac{1}{\left|\left(1+x^{*}\right) / 2-x\right|^{\alpha_{1} / 2}} \frac{\varphi^{-\alpha_{1}+k-1-\epsilon_{1}}(t)}{|x-t|^{k-\alpha_{1} / 2}} \\
& =C \varphi^{-\alpha_{1}}(x) \frac{1}{(1-t)^{\left(k-1+\varepsilon_{1}\right) / 2+1} \frac{\varphi^{-\alpha_{1}+2 k}(t)}{|x-t|^{k-\alpha_{1} / 2}}} \\
& =\varphi^{-\alpha_{1}}(x) O\left(n^{\max \{\alpha-2 k, 0\}}\right) \frac{1}{(1-t)^{\left(k-1+\varepsilon_{1}\right) / 2+1}},
\end{aligned}
$$

and we know that

$$
\int_{\left(1+x^{*}\right) / 2}^{1-C / n^{2}} \frac{1}{(1-t)^{\left(k-1+\varepsilon_{1}\right) / 2+1}} d t \leq C n^{k-1+\varepsilon_{1}} .
$$

Therefore, we see that

$$
\begin{aligned}
& \int_{\left(1+x^{*}\right) / 2}^{1-C / n^{2}} \frac{\varphi^{-\alpha_{1}+k-1-\epsilon_{1}}(t)}{|x-t|^{k}} d t \\
& \quad \leq C \varphi^{-\alpha_{1}}(x) n^{k-1+\varepsilon_{1}} O\left(n^{\max \left\{\alpha_{1}-2 k, 0\right\}}\right) .
\end{aligned}
$$

Consequently, we have the result (b).

(c) and (d): Since $\left|1-t^{2}\right| \leq C\left(\left|1-x^{2}\right|+|t-x|\right)$, we know $\varphi(t) \leq C\left(\varphi(x)+|x-t|^{1 / 2}\right)$. Then, we have

$$
\begin{aligned}
& \int_{\substack{0 \leq t \leq 1-C / n^{2},|x-t|>\varphi(x) / n}} \frac{\varphi^{\alpha+k-1}(t)}{|x-t|^{k}} d t \\
& \quad \leq C\left(\varphi^{\alpha+k-1}(x) \int \frac{1}{|x-t|^{k}} d t+\int \frac{1}{|x-t|^{(k+1) / 2}} d t\right) \\
& \quad \leq C n^{k-1} \begin{cases}1, & k \geqslant 2 ; \\
\ln n & k=1,\end{cases}
\end{aligned}
$$

because we see if $k=1$, then

$$
\begin{aligned}
& \int_{\substack{0 \leq t \leq 1-C / n^{2},|x-t|>\varphi(x) / n}} \frac{1}{|x-t|^{k}} d t \leq C \ln n, \\
& \int_{\substack{0 \leq t \leq 1-C / n^{2},|x-t|>\varphi(x) / n}} \frac{1}{|x-t|^{(k+1) / 2}} d t \leq C \ln n
\end{aligned}
$$

and if $k \geq 2$, then

$$
\begin{aligned}
& \varphi^{\alpha+k-1}(x) \int_{\substack{0 \leq t \leq 1-C / n^{2} \\
|x-t|>\varphi(x) / n}} \frac{1}{|x-t|^{k}} d t \\
& \quad \leq C \frac{\varphi^{\alpha+k-1}(x)}{|x-t|^{k-1}} \leq C \varphi^{\alpha+k-1}(x)\left(\frac{n}{\varphi(x)}\right)^{k-1} \\
& \quad \leq C n^{k-1},
\end{aligned}
$$

$$
\int_{\substack{0 \leq t \leq 1-C / n^{2} \\|x-t|>\varphi(x) / n}} \frac{1}{|x-t|^{(k+1) / 2}} d t \leq C n^{k-1}
$$

that is, we have (c). Similarly,

$$
\begin{aligned}
& \int_{\substack{0 \leq t \leq 1-C / n^{2},|x-t|>\varphi(x) / n}} \frac{\varphi^{\alpha_{1}+k-1-\epsilon_{1}}(t)}{|x-t|^{k}} d t \\
& \leq C\left(\varphi^{\alpha_{1}+k-1}(x) \int_{\substack{0 \leq t \leq 1-C / n^{2} \\
|x-t|>\varphi(x) / n}} \frac{\varphi^{-\epsilon_{1}}(t)}{|x-t|^{k}} d t\right. \\
& \left.\quad+\int_{\substack{0 \leq t \leq 1-C / n^{2} \\
|x-t|>\varphi(x) / n}} \frac{\varphi^{-\epsilon_{1}}(t)}{|x-t|^{(k+1) / 2}} d t\right) \\
& :=C(A+B) .
\end{aligned}
$$

Then, for $A$

$$
\begin{aligned}
A & =\varphi^{\alpha_{1}+k-1}(x) \int_{\substack{0 \leq t \leq 1-C / n^{2} \\
|x-t|>\varphi(x) / n}} \frac{\varphi^{-\epsilon_{1}}(t)}{|x-t|^{k}} d t \\
& \leq C \varphi^{\alpha_{1}+k-1}(x)\left(\frac{n}{\varphi(x)}\right)^{k-1} \int_{\substack{0 \leq t \leq 1-C / n^{2} \\
|x-t|>\varphi(x) / n}} \frac{\varphi^{-\epsilon_{1}}(t)}{|x-t|} d t \\
& \leq C n^{k-1} \int_{\substack{0 \leq t \leq 1-C / n^{2},|x-t|>\varphi(x) / n}} \frac{\varphi^{-\epsilon_{1}}(t)}{|x-t|} d t
\end{aligned}
$$

and for $B$

$$
\begin{aligned}
B & =\int_{\substack{0 \leq t \leq 1-C / n^{2},|x-t|>\varphi(x) / n}} \frac{\varphi^{-\epsilon_{1}}(t)}{|x-t|^{(k+1) / 2}} d t \\
& \leq C n^{k-1} \int_{\substack{0 \leq t \leq 1-C / n^{2},|x-t|>\varphi(x) / n}} \frac{\varphi^{-\epsilon_{1}}(t)}{|x-t|} d t .
\end{aligned}
$$

On the other hand, by (97) and (99), we know that

$$
\int_{0}^{x_{*}} \frac{\varphi^{-\epsilon_{1}}(t)}{x-t} d t \leq C n^{\varepsilon_{1}}, \quad \int_{x^{*}}^{\left(1+x^{*}\right) / 2} \frac{\varphi^{-\epsilon_{1}}(t)}{(t-x)} d t \leq C n^{\varepsilon_{1}}
$$

Since $|x-t| \geq C|1-t|$ for $\left(1+x^{*}\right) / 2<t<1-C / n^{2}$, we have

$$
\int_{\left(1+x^{*}\right) / 2}^{1-C / n^{2}} \frac{\varphi^{-\epsilon_{1}}(t)}{(t-x)} d t \leq C \int_{\left(1+x^{*}\right) / 2}^{1-C / n^{2}} \frac{1}{(1-t)^{\epsilon_{1} / 2+1}} d t \leq C n^{\varepsilon_{1}} .
$$

Therefore, we have

$$
\int_{\substack{0 \leq t \leq 1-C / n^{2} \\|x-t|>\varphi(x) / n}} \frac{\varphi^{-\epsilon_{1}}(t)}{|x-t|} d t \leq C n^{k-1+\varepsilon_{1}} .
$$

So, by (108) and (109) we have (d). 
Lemma 24. Let $0 \leq i \leq m-1$. Let $a>0$ be a fixed, sufficiently small constant. Let $x \in\left[\xi_{k-1, n+1}^{(\lambda)}, \xi_{k+1, n+1}^{(\lambda)}\right] \cap\left[0,1-a / n^{2}\right]$ for some $1 \leq k \leq n+1$. Then, one has uniformly for $n$

$$
\begin{aligned}
& \sum_{\substack{\mu \notin[k-2, k+2], 1 \leq \mu \leq n+1}} \frac{\varphi^{(1-\lambda) m}(x)}{n^{m-i}} \frac{\varphi^{\lambda m-i}\left(\xi_{\mu, n+1}^{(\lambda)}\right)}{\left|x-\xi_{\mu, n+1}^{(\lambda)}\right|^{m-i}} \\
& \quad \leq O\left(n^{\max \{(1-\lambda) m-2(m-i), 0\}}\right) \begin{cases}1, & 0 \leq i \leq m-2 ; \\
\ln n, & i=m-1 .\end{cases}
\end{aligned}
$$

Proof. For the simplicity, we denote

$$
\sum_{\mu \notin[k-2, k+2]}:=\sum_{\substack{\mu \notin[k-2, k+2] \\ 1 \leq \mu \leq n+1}} \frac{\varphi^{(1-\lambda) m}(x)}{n^{m-i}} \frac{\varphi^{\lambda m-i}\left(\xi_{\mu, n+1}^{(\lambda)}\right)}{\left|x-\xi_{\mu, n+1}^{(\lambda)}\right|^{m-i}} .
$$

Then, we know that

$$
\begin{aligned}
\sum_{\mu \notin[k-2, k+2]}= & \frac{\varphi^{(1-\lambda) m}(x)}{n^{m-i-1}} \\
& \times \sum_{\mu \notin[k-2, k+2]} \frac{\varphi\left(\xi_{\mu, n+1}^{(\lambda)}\right)}{n} \frac{\varphi^{(\lambda-1) m+m-i-1}\left(\xi_{\mu, n+1}^{(\lambda)}\right)}{\left|x-\xi_{\mu, n+1}^{(\lambda)}\right|^{m-i}} .
\end{aligned}
$$

Then, for some $C>0$ with $1-C / n^{2}>\left(1+\xi_{n+1, n+1}^{(\lambda)}\right) / 2$, we have by $(80)$

$$
\begin{aligned}
& \sum_{\mu \notin[k-2, k+2]} \frac{\varphi\left(\xi_{\mu, n+1}^{(\lambda)}\right)}{n} \frac{\varphi^{(\lambda-1) m+m-i-1}\left(\xi_{\mu, n+1}^{(\lambda)}\right)}{\left|x-\xi_{\mu, n+1}^{(\lambda)}\right|^{m-i}} \\
& \sim \int_{\left[-1+C / n^{2}, 1-C / n^{2}\right] \backslash\left[\xi_{k-2, n+1}^{(\lambda)} \xi_{k+2, n+1}^{(\lambda)}\right]} \frac{\varphi^{(\lambda-1) m+m-i-1}(t)}{|x-t|^{m-i}} d t .
\end{aligned}
$$

Therefore, we have the result from Lemma 23 (a) with $\alpha=$ $(1-\lambda) m, k=m-i$.

For convenience, we let

$$
H_{n+1, m, i}[f](x):=\sum_{\mu=1}^{n+1} e_{i, \mu} l_{\mu, n+1}^{m}(x)\left(x-\xi_{\mu, n+1}^{(\lambda)}\right)^{i} f\left(\xi_{\mu, n+1}^{(\lambda)}\right),
$$

then

$$
H_{n+1, m}[f](x):=\sum_{i=0}^{m-1} H_{n+1, m, i}[f](x) .
$$

Let

$$
\left\|H_{n+1, m, i}\right\|:=\sup _{x \in[-1,1]} \sum_{\mu=1}^{n+1}\left|e_{i, \mu} \mu_{\mu, n+1}^{m}(x)\left(x-\xi_{\mu, n+1}^{(\lambda)}\right)^{i}\right| .
$$

Lemma 25. Let $m \geq 2$ be a positive integer. Then, one has for $0 \leq i \leq m-1$

$$
\begin{aligned}
\left\|H_{n+1, m, i}\right\|= & O\left(n^{\max \{(1-\lambda) m-2(m-i), 0\}}\right) \\
& \times \begin{cases}1, & 0 \leq i \leq m-2 \\
\ln n, & i=m-1 .\end{cases}
\end{aligned}
$$

Proof. By [10, (26)], it is sufficient to prove (120) for $0 \leq x \leq$ $1-a / n^{2}$, where $a>0$ is a fixed, sufficiently small constant. In the following case, let $x \in\left[\xi_{k-1, n+1}^{(\lambda)}, \xi_{k+1, n+1}^{(\lambda)}\right] \cap\left[0,1-a / n^{2}\right]$ for some $1 \leq k \leq n+1$. Then, we have from (32), (34), and (47)

$$
\begin{aligned}
& \sum_{\mu \notin[k-2, k+2]}\left|e_{i, \mu} l_{\mu, n+1}^{m}(x)\left(x-\xi_{\mu, n+1}^{(\lambda)}\right)^{i}\right| \\
= & \sum_{\mu \notin[k-2, k+2]}\left|e_{i, \mu}\right|\left|\frac{E_{\lambda, n+1}(x)}{E_{\lambda, n+1}^{\prime}\left(\xi_{\mu, n+1}^{(\lambda)}\right)}\right|^{m} \frac{1}{\left|x-\xi_{\mu, n+1}^{(\lambda)}\right|^{m-i}} \\
\leq & C \sum_{\mu \notin[k-2, k+2]} \frac{n^{i}}{\varphi^{i}\left(\xi_{\mu, n+1}^{(\lambda)}\right)}\left|\frac{n^{1-\lambda} \varphi^{1-\lambda}(x)}{n^{2-\lambda} \varphi^{-\lambda}\left(\xi_{\mu, n+1}^{(\lambda)}\right)}\right|^{m} \frac{1}{\left|x-\xi_{\mu, n+1}^{(\lambda)}\right|^{m-i}} \\
\leq & C \frac{\varphi^{(1-\lambda) m}(x)}{n^{m-i}} \sum_{\mu \notin[k-2, k+2]} \frac{\varphi^{\lambda m-i}\left(\xi_{\mu, n+1}^{(\lambda)}\right)}{\left|x-\xi_{\mu, n+1}^{(\lambda)}\right|^{m-i}} \\
= & O\left(n^{\max \{(1-\lambda) m-2(m-i), 0\}}\right) \begin{cases}1, & 0 \leq i \leq m-2 ; \\
\ln n, & i=m-1 .\end{cases}
\end{aligned}
$$

The last inequality follows from Lemma 24 . On the other hand, if $\mu \in[k-2, k+2]$, there exists $\theta_{\mu}$ between $x$ and $\xi_{\mu, n+1}^{(\lambda)}$ such that we see from (34) and (36) with $r=1$,

$$
\left|l_{\mu, n+1}(x)\right|=\left|\frac{E_{\lambda, n+1}^{\prime}\left(\theta_{\mu}\right)}{E_{\lambda, n+1}^{\prime}\left(\xi_{\mu, n+1}^{(\lambda)}\right)}\right| \leq C
$$

Hence, from (47) and (80), we conclude that

$$
\sum_{\substack{\mu \in[k-2, k+2] \\ 1 \leq \mu \leq n+1}}\left|e_{i, \mu} l_{\mu, n+1}^{m}(x)\left(x-\xi_{\mu, n+1}^{(\lambda)}\right)^{i}\right| \leq C .
$$

Therefore, we have the result.

Lemma 26. Let $m \geq 2$ be an even integer. Then,

$$
\left\|H_{n+1, m, m-1}\right\|=O\left(n^{\max \{(1-\lambda) m-2,0\}}\right) .
$$

Proof. First, we note that $m-1$ is odd. Let $x \in$ $\left[\xi_{k-1, n+1}^{(\lambda)}, \xi_{k+1, n+1}^{(\lambda)}\right] \cap\left[0,1-a / n^{2}\right]\left(\xi_{0, n+1}^{(\lambda)}:=-1, \xi_{n+2, n+1}^{(\lambda)}:=1\right)$ 
for some $1 \leq \mu \leq n+1$. Then, considering $\alpha_{1}, \varepsilon_{1}$ as $(1-\lambda) m$, $1-\lambda$, we have from (32), (34), (48), and Lemma 23(b)

$$
\begin{aligned}
& \sum_{\mu \notin[k-2, k+2]}\left|e_{m-1, \mu} l_{\mu, n+1}^{m}(x)\left(x-\xi_{\mu, n+1}^{(\lambda)}\right)^{m-1}\right| \\
= & \sum_{\mu \notin[k-2, k+2]}\left|e_{m-1, \mu}\right|\left|\frac{E_{\lambda, n+1}(x)}{E_{\lambda, n+1}^{\prime}\left(\xi_{\mu, n+1}^{(\lambda)}\right)}\right|^{m} \frac{1}{\left|x-\xi_{\mu, n+1}^{(\lambda)}\right|} \\
\leq & C \frac{\varphi^{(1-\lambda) m}(x)}{n^{2-\lambda}} \sum_{\mu \notin[k-2, k+2]} \frac{\varphi^{\lambda m-m+1-(1-\lambda)}\left(\xi_{\mu, n+1}^{(\lambda)}\right)}{\left|x-\xi_{\mu, n+1}^{(\lambda)}\right|} \\
= & \frac{\varphi^{(1-\lambda) m}(x)}{n^{1-\lambda}} \sum_{\mu \notin[k-2, k+2]} \frac{\varphi\left(\xi_{\mu, n+1}^{(\lambda)}\right)}{n} \frac{\varphi^{(\lambda-1) m-(1-\lambda)}\left(\xi_{\mu, n+1}^{(\lambda)}\right)}{\left|x-\xi_{\mu, n+1}^{(\lambda)}\right|} \\
\sim & \frac{\varphi^{(1-\lambda) m}(x)}{n^{1-\lambda}} \int \frac{\varphi^{(\lambda-1) m-(1-\lambda)}(t)}{|x-t|} d t=O\left(n^{\max \{(1-\lambda) m-2,0\}}\right),
\end{aligned}
$$

where it is integrated under $\left[-1+C / n^{2}, 1-C / n^{2}\right] ।$ $\left[\xi_{k-2, n+1}^{(\lambda)}, \xi_{k+2, n+1}^{(\lambda)}\right]$. Besides, we have, similarly to (123),

$$
\sum_{\mu \in[k-2, k+2]}\left|e_{m-1, \mu} l_{\mu, n+1}^{m}(x)\left(x-\xi_{\mu, n+1}^{(\lambda)}\right)^{i}\right| \leq C .
$$

Therefore, we have the result.

Lemma 27. For any polynomial $R \in \mathscr{P}_{n m+m-1}$, one has

$$
\begin{aligned}
& \left|R(x)-H_{n+1, m}[R](x)\right| \\
& \quad \leq C \sum_{t=1}^{m-1} \sum_{i=0}^{m-1-t} \frac{\left\|R^{(t)} \varphi^{t}\right\|_{\infty}}{n^{t}} O\left(n^{\max \{(1-\lambda) m-2(m-t-i), 0\}}\right) \ln n .
\end{aligned}
$$

Proof. Let $x \in\left[\xi_{k-1, n+1}^{(\lambda)}, \xi_{k+1, n+1}^{(\lambda)}\right] \cap\left[0,1-a / n^{2}\right]\left(\xi_{0, n+1}^{(\lambda)}:=\right.$ $\left.-1, \xi_{n+2, n+1}^{(\lambda)}:=1\right)$ for some $1 \leq \mu \leq n+1$.

$$
\begin{aligned}
& R(x)-H_{n+1, m}[R](x) \\
& =\widehat{H}_{n+1, m}[R](x)-H_{n+1, m}[R](x) \\
& =\sum_{t=1}^{m-1} \sum_{\mu=1}^{n+1} R^{(t)}\left(\xi_{\mu, n+1}^{(\lambda)}\right) h_{t \mu}(x) \\
& =\sum_{\mu=1}^{n+1} \sum_{t=1}^{m-1} \sum_{i=0}^{m-1-t} \frac{e_{t, i, \mu}}{t !} R^{(t)}\left(\xi_{\mu, n+1}^{(\lambda)}\right) l_{\mu, n+1}^{m}(x)\left(x-\xi_{\mu, n+1}^{(\lambda)}\right)^{t+i} \\
& =\sum_{\mu \notin[k-2, k+2]}+\sum_{\mu \in[k-2, k+2]} .
\end{aligned}
$$

Here, $e_{t, i, k}$ is the coefficient of the higher-order Hermite interpolation polynomial $\widehat{H}_{n+1, m}[R]$ based on the zeros of
$E_{\lambda, n+1}$, defined in (11). Since $e_{t, i, k}=e_{0, i, k}=e_{i, k}$, we can see from (47) that uniformly for $0 \leq i \leq m-1$,

$$
\left|e_{t, i, \mu}\right| \leq \operatorname{Cn}^{i} \varphi^{-i}\left(\xi_{\mu, n+1}^{(\lambda)}\right)
$$

Hence, using (32) and (34), we see for $\mu \notin[k-2, k+2]$

$$
\begin{aligned}
& \left|\frac{e_{t, i, \mu}}{t !} R^{(t)}\left(\xi_{\mu, n+1}^{(\lambda)}\right) l_{\mu, n+1}^{m}(x)\left(x-\xi_{\mu, n+1}^{(\lambda)}\right)^{t+i}\right| \\
& \leq C \frac{\left\|R^{(t)} \varphi^{t}\right\|_{\infty}}{n^{t}} \frac{\varphi^{(1-\lambda) m}(x)}{n^{m-(t+i)}} \frac{\varphi^{\lambda m-t-i}\left(\xi_{\mu, n+1}^{(\lambda)}\right)}{\left|x-\xi_{\mu, n+1}^{(\lambda)}\right|^{m-(t+i)}}
\end{aligned}
$$

Here, using Lemma 24 with $t+i$ as $i$ for $\mu \notin[k-2, k+2]$, we have the right formula in the lemma. We also see that for $\mu \in[k-2, k+2]$

$$
\left|\frac{e_{t, i, \mu}}{t !} R^{(t)}\left(\xi_{\mu, n+1}^{(\lambda)}\right) l_{\mu, n+1}^{m}(x)\left(x-\xi_{\mu, n+1}^{(\lambda)}\right)^{t+i}\right| \leq C \frac{\left\|R^{(t)} \varphi^{t}\right\|_{\infty}}{n^{t}} .
$$

Consequently, we have

$$
\begin{aligned}
& \left|R(x)-H_{n+1, m}[R](x)\right| \\
& \quad \leq C \sum_{t=1}^{m-1} \sum_{i=0}^{m-1-t} \frac{\left\|R^{(t)} \varphi^{t}\right\|_{\infty}}{n^{t}} O\left(n^{\max \{(1-\lambda) m-2(m-t-i), 0\}}\right) \ln n .
\end{aligned}
$$

Proof of Theorem 1. (a) From Lemma 26, the result is trivially proved.

(b) Since $f$ is continuous on $[-1,1]$, for given $\varepsilon>0$, there exists a polynomial $R$ such that for $x \in[-1,1]$

$$
|f(x)-R(x)|<\varepsilon
$$

Then, one has from Lemmas 26 and 27

$$
\begin{aligned}
\lim _{n \rightarrow \infty} & \left|H_{n+1, m}[f](x)-f(x)\right| \\
\leq & |f(x)-R(x)|+\lim _{n \rightarrow \infty}\left|H_{n+1, m}[f-R](x)\right| \\
& +\lim _{n \rightarrow \infty}\left|H_{n+1, m}[R](x)-R(x)\right| \\
\leq & C\left(1+\left\|H_{n+1, m}\right\|\right)\|f-R\|_{\infty} \\
& +\lim _{n \rightarrow \infty} \sum_{t=1}^{m-1} \sum_{i=0}^{m-1-t} \frac{\left\|R^{(t)} \varphi^{t}\right\|_{\infty}}{n^{t}} \ln n \leq C \varepsilon .
\end{aligned}
$$

This implies (21).

Lemma 28. Let $0 \leq i \leq m-1$. Let $a>0$ be a fixed, sufficiently small constant. $x \in\left[y_{k-1,2 n+1}^{(\lambda)}, y_{k+1,2 n+1}^{(\lambda)}\right] \cap[0,1-$ 
$\left.a / n^{2}\right]\left(y_{0,2 n+1}^{(\lambda)}:=-1, y_{2 n+2,2 n+1}^{(\lambda)}:=1\right)$ for some $1 \leq k \leq 2 n+1$. Then we have uniformly for $n$

$$
\begin{gathered}
\sum_{\substack{\nu \notin[k-2, k+2], 1 \leq \nu \leq 2 n+1}} \frac{\varphi^{(1-2 \lambda) m}(x)}{n^{m-i}} \frac{\varphi^{2 \lambda m-i}\left(y_{\nu, 2 n+1}^{(\lambda)}\right)}{\left|x-y_{\nu, 2 n+1}^{(\lambda)}\right|^{m-i}} \\
\leq C \begin{cases}A_{n, i}(x) & \text { if } 0<\lambda<\frac{1}{2} ; \\
B_{n, i}(x) & \text { if } \frac{1}{2} \leq \lambda<1,\end{cases}
\end{gathered}
$$

where

$$
\begin{aligned}
A_{n, i}(x)= & n^{\max \{(1-2 \lambda) m-2(m-i), 0\}} \\
& \times \begin{cases}1, & 0 \leq i \leq m-2 ; \\
\ln n, & i=m-1,\end{cases} \\
B_{n, i}(x)= & \varphi^{(1-2 \lambda) m}(x) \\
& \times \begin{cases}1, & 0 \leq i \leq m-2 ; \\
\ln n, & i=m-1\end{cases}
\end{aligned}
$$

Proof. When $0<\lambda<1 / 2$, we can show the lemma as the proof of Lemma 24 (see (116)). If $1 / 2 \leq \lambda<1$, we have from Lemma 23 (c),

$$
\begin{aligned}
& \sum_{\substack{v \notin[k-2, k+2], 1 \leq \nu \leq 2 n+1}} \leq C \frac{\varphi^{(1-2 \lambda) m}(x)}{n^{m-i-1}} \\
& \quad \times \int_{\left.\left[-1+a / n^{2}, 1-a / n^{2}\right] \backslash y_{k-1,2 n+1}^{(\lambda)}, y_{k+1,2 n+1}^{(\lambda)}\right]} \frac{\varphi^{(2 \lambda-1) m+m-i-1}(t)}{|x-t|^{m-i}} d t \\
& \leq C\left\{\begin{array}{l}
A_{n, i}(x) \quad \text { if } 0<\lambda<\frac{1}{2} ; \\
B_{n, i}(x) \quad \text { if } \frac{1}{2} \leq \lambda<1 .
\end{array}\right.
\end{aligned}
$$

Let

$$
\begin{aligned}
\mathscr{H}_{2 n+1, m, i}[f](x):= & \sum_{\nu=1}^{2 n+1} e_{i, v}^{*} l_{\nu, 2 n+1}^{* m}(x) \\
& \times\left(x-y_{v, 2 n+1}^{(\lambda)}\right)^{i} f\left(y_{\nu, 2 n+1}^{(\lambda)}\right),
\end{aligned}
$$

then

$$
\mathscr{H}_{2 n+1, m}[f](x):=\sum_{i=0}^{m-1} \mathscr{H}_{2 n+1, m, i}[f](x)
$$

Let

$$
\left\|\mathscr{H}_{2 n+1, m, i}\right\|:=\sup _{x \in[-1,1]} \sum_{\nu=1}^{2 n+1}\left|e_{i, v}^{*} l_{v, 2 n+1}^{* m}(x)\left(x-y_{v, 2 n+1}^{(\lambda)}\right)^{i}\right|
$$

and for a nonnegative real function $u(x)$,

$$
\begin{aligned}
\left\|\mathscr{H}_{2 n+1, m, i}\right\|_{u}:=\sup _{x \in[-1,1]} u(x) \\
\quad \times \sum_{\nu=1}^{2 n+1}\left|e_{i, \nu}^{*} l_{\nu, 2 n+1}^{* m}(x)\left(x-y_{\nu, 2 n+1}^{(\lambda)}\right)^{i}\right| .
\end{aligned}
$$

Lemma 29. Let $m \geq 2$ be a positive integer. If $0<\lambda<1 / 2$, then one has for $0 \leq i \leq m-1$

$$
\left\|\mathscr{H}_{2 n+1, m, i}\right\|=O\left(n^{\max \{(1-2 \lambda) m-2(m-i), 0\}}\right)
$$

$$
\times \begin{cases}1, & 0 \leq i \leq m-2 \\ \ln n, & i=m-1\end{cases}
$$

If $1 / 2 \leq \lambda<1$, then one has for $0 \leq i \leq m-1$

$$
\begin{aligned}
\left\|\mathscr{H}_{2 n+1, m, i}\right\|_{\varphi^{(2 \lambda-1) m}}= & O(1) \\
& \times \begin{cases}1, & 0 \leq i \leq m-2 ; \\
\ln n, & i=m-1 .\end{cases}
\end{aligned}
$$

Proof. Similarly to the proof of Lemma 25, using (51), we have from Proposition 7(2), Proposition 8 (35), and Lemma 28

$$
\begin{aligned}
& \sum_{\substack{v \notin[k-2, k+2], 1 \leq \nu \leq 2 n+1}}\left|e_{i, \nu}^{*} l_{\nu, 2 n+1}^{* m}(x)\left(x-y_{\nu, 2 n+1}^{(\lambda)}\right)^{i}\right| \\
& \leq C \sum_{\substack{\nu \notin[k-2, k+2], 1 \leq \nu \leq 2 n+1}} \frac{\varphi^{(1-2 \lambda) m}(x)}{n^{m-i}} \frac{\varphi^{2 \lambda m-i}\left(y_{v, 2 n+1}^{(\lambda)}\right)}{\left|x-y_{\nu, 2 n+1}^{(\lambda)}\right|^{m-i}} \\
& \leq C \begin{cases}A_{n, i}(x) & \text { if } 0<\lambda<\frac{1}{2} ; \\
B_{n, i}(x) & \text { if } \frac{1}{2} \leq \lambda<1,\end{cases}
\end{aligned}
$$

where $A_{n, i}(x)$ and $B_{n, i}(x)$ are defined by (136) or (137). Besides, we easily know from (51), (35), (38), and (80)

$$
\sum_{\substack{v \in[k-2, k+2], 1 \leq \nu \leq 2 n+1}}\left|e_{i, \nu}^{*} l_{\nu, 2 n+1}^{* m}(x)\left(x-y_{\nu, 2 n+1}^{(\lambda)}\right)^{i}\right| \leq C .
$$

Therefore, we have the result.

Let

$$
\begin{aligned}
\mathscr{H}_{2 n+1, m, i}[f](x):=\sum_{\nu=1}^{2 n+1} e_{i, \nu}^{*} l_{v, 2 n+1}^{* m}(x) & \\
& \times\left(x-y_{v, 2 n+1}^{(\lambda)}\right)^{i} f\left(y_{v, 2 n+1}^{(\lambda)}\right),
\end{aligned}
$$

then

$$
\mathscr{H}_{2 n+1, m}[f](x):=\sum_{i=0}^{m-1} \mathscr{H}_{2 n+1, m, i}[f](x) .
$$

Let

$$
\left\|\mathscr{H}_{2 n+1, m, i}\right\|:=\sup _{x \in[-1,1]} \sum_{\nu=1}^{2 n+1}\left|e_{i, v}^{*} l_{v, 2 n+1}^{* m}(x)\left(x-y_{v, 2 n+1}^{(\lambda)}\right)^{i}\right| .
$$


Lemma 30. Let $m \geq 2$ be an even integer. Then, one has for $0<\lambda<1 / 2$

$$
\left\|\mathscr{H}_{2 n+1, m, m-1}\right\|=O\left(n^{\max \{(1-2 \lambda) m-2,0\}}\right)
$$

and for $1 / 2 \leq \lambda<1$

$$
\left\|\mathscr{H}_{2 n+1, m, m-1}\right\|_{\varphi^{(2 \lambda-1) m}}=O(1) .
$$

Proof. First, we note that $m-1$ is odd. Similarly to the proof of Lemma 26, using Proposition 7(2), Proposition 8 (35), and (51), we have

$$
\begin{aligned}
\sum:= & \sum_{\substack{\nu \notin[k-2, k+2], 1 \leq \nu \leq 2 n+1}}\left|e_{m-1, \nu}^{*} l_{\nu, 2 n+1}^{* m}(x)\left(x-y_{\nu, 2 n+1}^{(\lambda)}\right)^{m-1}\right| \\
\leq & C \frac{\varphi^{(1-2 \lambda) m}(x)}{n^{2-\lambda}} \\
& \times \sum_{\nu \notin[k-2, k+2],} \frac{\varphi^{2 \lambda m-m+1-(1-\lambda)}\left(y_{\nu, 2 n+1}^{(\lambda)}\right)}{\left|x-y_{\nu, 2 n+1}^{(\lambda)}\right|} \\
\leq & C \frac{\varphi^{(1-2 \lambda) m}(x)}{n^{1-\lambda}} \int_{A} \frac{\varphi^{(2 \lambda-1) m-(1-\lambda)}(t)}{|x-t|} d t,
\end{aligned}
$$

where $A:=\left[-1+a / n^{2}, 1-a / n^{2}\right] \backslash\left[y_{k-1,2 n+1}^{(\lambda)}, y_{k+1,2 n+1}^{(\lambda)}\right]$. If $0<\lambda<1 / 2$, considering $\alpha_{1}$ and $\varepsilon_{1}$ as $(1-2 \lambda) m$ and $(1-\lambda)$, respectively, we have from Lemma 23(b)

$$
\begin{aligned}
\int_{A} \frac{\varphi^{(2 \lambda-1) m-(1-\lambda)}(t)}{|x-t|} d t \leq & C \varphi^{(2 \lambda-1) m}(x) n^{1-\lambda} \\
& \times O\left(n^{\max \{(1-2 \lambda) m-2,0\}}\right)
\end{aligned}
$$

and if $1 / 2 \leq \lambda<1$, considering $\alpha_{1}$ and $\varepsilon_{1}$ as $(2 \lambda-1) m$ and $(1-\lambda)$, respectively, we have from Lemma 23(d)

$$
\int_{A} \frac{\varphi^{(2 \lambda-1) m-(1-\lambda)}(t)}{|x-t|} d t \leq C n^{1-\lambda}
$$

Hence, we conclude that

$$
\sum \leq C \begin{cases}n^{\max \{(1-2 \lambda) m-2,0\}}, & 0<\lambda<\frac{1}{2} \\ \varphi^{(1-2 \lambda) m}(x), & \frac{1}{2} \leq \lambda<1 .\end{cases}
$$

Besides, we easily know that

$$
\sum_{\substack{v \in[k-2, k+2], 1 \leq \nu \leq 2 n+1}}\left|e_{m-1, v}^{*} l_{v, 2 n+1}^{* m}(x)\left(x-y_{v, 2 n+1}^{(\lambda)}\right)^{m-1}\right| \leq C .
$$

Therefore, we have the result.
Lemma 31. For any polynomial $R \in \mathscr{P}_{2 n m+m-1}$, one has

$$
\begin{aligned}
& \left|R(x)-\mathscr{H}_{2 n+1, m}[R](x)\right| \\
& \leq C \sum_{t=1}^{m-1} \sum_{i=0}^{m-1-t} \frac{\left\|R^{(t)} \varphi^{t}\right\|_{\infty}}{n^{t}} \\
& \quad \times \begin{cases}A_{n, t+i}(x) \quad \text { if } 0<\lambda<\frac{1}{2} ; \\
B_{n, t+i}(x) \quad \text { if } \frac{1}{2} \leq \lambda<1 .\end{cases}
\end{aligned}
$$

Proof. We have using Lemma 28

$$
\begin{aligned}
& \left|R(x)-\mathscr{H}_{2 n+1, m}[R](x)\right| \\
& \leq C \sum_{t=1}^{m-1} \sum_{i=0}^{m-1-t 2 n+1} \sum_{v=1} \frac{\left|R^{(t)}\left(y_{v, 2 n+1}^{(\lambda)}\right) \varphi^{t}\left(y_{v, 2 n+1}^{(\lambda)}\right)\right|}{n^{t}} \\
& \times \frac{\varphi^{(1-2 \lambda) m}(x)}{n^{m-(t+i)}} \frac{\varphi^{2 \lambda m-t-i}\left(y_{v, 2 n+1}^{(\lambda)}\right)}{\left|x-y_{v, 2 n+1}^{(\lambda)}\right|^{m-(t+i)}} \\
& \leq C \sum_{t=1}^{m-1} \sum_{i=0}^{m-1-t} \frac{\left\|R^{(t)} \varphi^{t}\right\|_{\infty}}{n^{t}}\left\{\begin{array}{l}
A_{n, t+i}(x), \quad \text { if } 0<\lambda<\frac{1}{2} ; \\
B_{n, t+i}(x), \quad \text { if } \frac{1}{2} \leq \lambda<1 .
\end{array}\right.
\end{aligned}
$$

Proof of Theorem 2. Using Lemmas 29, 30, and 31, it is similar to the proof of Theorem 1 .

\subsection{Proof of Theorems 3 and 4}

Proof of Theorems 3 and 4. Suppose that $m=1,3,5, \ldots$, and let

$$
\Lambda_{n+1, m}(x):=\sum_{\mu=1}^{n+1} \sum_{i=0}^{m-1}\left|e_{i, \mu} l_{\mu, n+1}^{m}(x)\left(x-\xi_{\mu, n+1}^{(\lambda)}\right)^{i}\right| .
$$

From (17), we know that

$$
\left\|H_{n+1, m}\right\|=\sup _{x \in[-1,1]} \Lambda_{n+1, m}(x) .
$$

Let $x_{0}$ be the least positive zero of $P_{\lambda, n}(x)$. Then, we have

$$
\begin{aligned}
& \Lambda_{n+1, m}\left(x_{0}\right) \\
& \geq \sum_{-1 / 2 \leq \xi_{\mu, n+1}^{(\lambda)} \leq 0}\left|\frac{E_{\lambda, n+1}\left(x_{0}\right)}{E_{\lambda, n+1}^{\prime}\left(\xi_{\mu, n+1}^{(\lambda)}\right)}\right|^{m} \\
& \quad \times\left(\frac{\left|e_{m-1, \mu}\right|}{x_{0}-\xi_{\mu, n+1}^{(\lambda)}}-\sum_{i=0}^{m-2} \frac{\left|e_{i, \mu}\right|}{\left(x_{0}-\xi_{\mu, n+1}^{(\lambda)}\right)^{m-i}}\right) .
\end{aligned}
$$


Since we know from (42), (34), and (47)

$$
\begin{aligned}
& \sum_{-1 / 2 \leq \xi_{\mu, n+1}^{(\lambda)} \leq 0}\left|\frac{E_{\lambda, n+1}\left(x_{0}\right)}{E_{\lambda, n+1}^{\prime}\left(\xi_{\mu, n+1}^{(\lambda)}\right)}\right|^{m} \frac{\left|e_{m-1, \mu}\right|}{x_{0}-\xi_{\mu, n+1}^{(\lambda)}} \\
& \sim \sum_{-1 / 2 \leq \xi_{\mu, n+1}^{(\lambda)} \leq 0} \frac{1}{n} \frac{1}{x_{0}-\xi_{\mu, n+1}^{(\lambda)}} \sim \int_{-1 / 2}^{0} \frac{1}{x_{0}-t} d t \sim \ln n, \\
& -1 / 2 \leq \xi_{\mu, n+1}^{(\lambda)} \leq 0 \\
& \left.\sim \sum_{i=0}^{m-2} \frac{1}{E_{\lambda, n+1}^{\prime}\left(\xi_{\mu, n+1}^{(\lambda)}\right)}\right|^{m-i-1} \frac{\left|e_{i, \mu}\right|}{\sum_{i=0}^{m-2} \frac{\left(x_{0}-\xi_{\mu, n+1}^{(\lambda)}\right)^{m-i}}{-1 / 2 \leq \xi_{\mu, n+1}^{(\lambda)} \leq 0}} \frac{1}{n} \frac{1}{\left(x_{0}-\xi_{\mu, n+1}^{(\lambda)}\right)^{m-i}} \\
& \sim \sum_{i=0}^{m-2} \frac{1}{n^{m-i-1}} \int_{-1 / 2}^{0} \frac{1}{\left(x_{0}-t\right)^{m-i}} d t \sim 1 .
\end{aligned}
$$

Thus, we have

$$
\Lambda_{n+1, m}\left(x_{0}\right) \geq C \ln n \text {. }
$$

Therefore, we have the result from Lemma 29.

Remark 32. Similarly, if we let $x_{T}$ be the least zero of $P_{\lambda, n}(x)$ with $x_{T}>c$ and if we consider $\Lambda_{n+1, m}(x)$ for $(-1+c) / 2 \leq$ $\xi_{\mu, n+1}^{(\lambda)} \leq c$, then we have for $-1<c<d<1$

$$
\max _{[c, d]} \Lambda_{n+1, m}(x) \geq C \ln n .
$$

Proof of Theorem 4. Suppose that $m=2,4, \ldots$.. Let $x_{0}$ be the least positive zero of $P_{\lambda, n}(x)$. Then, similarly to the proof of Theorem 3, we have using the assumption (27)

$$
\begin{aligned}
& \Lambda_{n+1, m}\left(x_{0}\right) \\
& \geq \sum_{-1 \leq \xi_{\mu, n+1}^{(\lambda)} \leq-1 / 2}\left|\frac{E_{\lambda, n+1}\left(x_{0}\right)}{E_{\lambda, n+1}^{\prime}\left(\xi_{\mu, n+1}^{(\lambda)}\right)}\right|^{m}\left(\left|e_{m-1, \mu}\right|-\sum_{i=0}^{m-2}\left|e_{i, \mu}\right|\right) \\
& \geq C \sum_{-1 \leq \xi_{\mu, n+1}^{(\lambda)} \leq-1 / 2} \frac{\varphi\left(\xi_{\mu, n+1}^{(\lambda)}\right)}{n} n^{\lambda-1} \varphi^{(\lambda-1)(m+1)}\left(\xi_{\mu, n+1}^{(\lambda)}\right) \\
& \sim n^{\lambda-1} \int_{-1+1 / n^{2}}^{-1 / 2} \varphi^{(\lambda-1)(m+1)}(t) d t \sim n^{(1-\lambda) m-2} .
\end{aligned}
$$

Here, we used the followings:

$$
\begin{gathered}
\frac{n^{\lambda+m-2}}{\varphi^{-\lambda+m}\left(\xi_{\mu, n+1}^{(\lambda)}\right)}-C_{1} \sum_{i=0}^{m-2} \frac{n^{i}}{\varphi^{i}\left(\xi_{\mu, n+1}^{(\lambda)}\right)} \geq C_{2} \frac{n^{\lambda+m-2}}{\varphi^{-\lambda+m}\left(\xi_{\mu, n+1}^{(\lambda)}\right)} \\
\int_{-1+1 / n^{2}}^{-1 / 2} \varphi^{(\lambda-1)(m+1)}(t) d t \sim n^{(1-\lambda) m-1-\lambda}
\end{gathered}
$$

because $((\lambda-1) m+\lambda-1) / 2<(-3+\lambda) / 2<-1$. Thus, we have $\left\|H_{n+1, m}\right\| \geq C n^{(1-\lambda) m-2}$, and it implies (28) from (20).
Proposition 33 (see $[6,(8.21 .10)])$. For $\varepsilon \leq \theta \leq \pi-\varepsilon$,

$$
\begin{aligned}
P_{\lambda, n}(\cos \theta)= & \frac{\Gamma(\lambda+1 / 2)}{\Gamma(2 \lambda)} n^{\lambda-1} \pi^{-1 / 2} 2^{\lambda} \sin ^{-\lambda} \theta \\
& \times \cos \left\{(n+\lambda) \theta-\frac{\lambda \pi}{2}\right\}+O\left(n^{\lambda-2}\right) .
\end{aligned}
$$

Proposition 34 (see [11, Theorem]). For $\varepsilon \leq \theta \leq \pi-\varepsilon$,

$$
\begin{aligned}
E_{\lambda, n+1}(\cos \theta)= & n^{1-\lambda} \pi^{-1 / 2} 2^{2-\lambda} \sin ^{1-\lambda} \theta \\
& \times \cos \left\{(n+\lambda) \theta-\frac{(\lambda-1) \pi}{2}\right\}+o\left(n^{1-\lambda}\right) .
\end{aligned}
$$

Lemma 35. For $\varepsilon \leq \theta \leq \pi-\varepsilon$,

$$
\begin{aligned}
F_{\lambda, 2 n+1}(\cos \theta)= & -2 \frac{\Gamma(\lambda+1 / 2)}{\Gamma(2 \lambda)} \\
& \times \pi^{-1} \sin ^{1-2 \lambda} \theta \sin \{2(n+\lambda) \theta-\lambda \pi\}+o(1) .
\end{aligned}
$$

Proof. It is proved from (167) and (168).

Proof of Theorem 5. Suppose that $m=1,3,5, \ldots$, and let

$$
\widetilde{\Lambda}_{2 n+1, m}(x):=\sum_{\nu=1}^{2 n+1} \sum_{i=0}^{m-1}\left|e_{i, \nu}^{*} l_{v, 2 n+1}^{* m}(x)\left(x-y_{v, 2 n+1}^{(\lambda)}\right)^{i}\right| .
$$

From (18), we know that

$$
\left\|\mathscr{H}_{2 n+1, m}\right\|=\sup _{x \in[-1,1]} \widetilde{\Lambda}_{2 n+1, m}(x) .
$$

Let $x_{0}:=\cos ((\lambda+n+1 / 2) \pi / 2(n+\lambda))$. Then, we know $F_{v, 2 n+1}\left(x_{0}\right) \sim 1$ from (169). Then, similarly to the proof of Theorem 3, we have from (35), (56), and (51), for $x>0$,

$$
\begin{aligned}
& \tilde{\Lambda}_{2 n+1, m}\left(x_{0}\right) \\
& \geq \sum_{-1 / 2 \leq y_{v, 2 n+1}^{(\lambda)} \leq 0}\left|\frac{F_{v, 2 n+1}\left(x_{0}\right)}{F_{v, 2 n+1}^{\prime}\left(y_{v, 2 n+1}^{(\lambda)}\right)}\right|^{m} \\
& \quad \times\left(\frac{\left|e_{m-1, v}^{*}\right|}{x_{0}-y_{v, 2 n+1}^{(\lambda)}}-\sum_{i=0}^{m-2} \frac{\left|e_{i, v}^{*}\right|}{\left(x_{0}-y_{v, 2 n+1}^{(\lambda)}\right)^{m-i}}\right)
\end{aligned}
$$$$
\geq C \ln n \text {. }
$$

Therefore, we have the result from Lemma 29.

Proof of Theorem 6. Suppose that $m=2,4, \ldots$ Let $x_{0}:=$ $\cos ((\lambda+n+1 / 2) \pi / 2(n+\lambda))$. Then, since we know that 
$F_{v, 2 n+1}\left(x_{0}\right) \sim 1$ as we see above, similarly to the proof of Theorem 4, we have using the assumption (30)

$$
\begin{aligned}
& \tilde{\Lambda}_{2 n+1, m}\left(x_{0}\right) \\
& \geq \sum_{-1 \leq y_{v, 2 n+1}^{(\lambda)} \leq-1 / 2}\left|\frac{F_{v, 2 n+1}\left(x_{0}\right)}{F_{v, 2 n+1}^{\prime}\left(y_{v, 2 n+1}^{(\lambda)}\right)}\right|^{m} \\
& \quad \times\left(\left|e_{m-1, \nu}^{*}\right|-\sum_{i=0}^{m-2}\left|e_{i, \nu}^{*}\right|\right) \\
& \geq C \sum_{-1 \leq y_{v, 2 n+1}^{(\lambda)} \leq-1 / 2}\left(\frac{\varphi^{2 \lambda}\left(y_{v, 2 n+1}^{(\lambda)}\right)}{n}\right)^{m} \frac{n^{\lambda+m-2}}{\varphi^{-\lambda+m}\left(y_{v, 2 n+1}^{(\lambda)}\right)} \\
& \sim n^{\lambda-1} \int_{-1+1 / n^{2}}^{-1 / 2} \varphi^{(2 \lambda-1) m+(\lambda-1)}(t) d t \sim n^{(1-2 \lambda) m-2} .
\end{aligned}
$$

Here, we used the following:

$$
\begin{gathered}
\left(\frac{\varphi^{2 \lambda}\left(y_{v, 2 n+1}^{(\lambda)}\right)}{n}\right)^{m}\left(\frac{n^{\lambda+m-2}}{\varphi^{-\lambda+m}\left(y_{v, 2 n+1}^{(\lambda)}\right)}-C_{1} \frac{n^{m-2}}{\varphi^{m-2}\left(y_{v, 2 n+1}^{(\lambda)}\right)}\right) \\
\geq C_{2}\left(\frac{\varphi^{2 \lambda}\left(y_{v, 2 n+1}^{(\lambda)}\right)}{n}\right)^{m} \frac{n^{\lambda+m-2}}{\varphi^{-\lambda+m}\left(y_{v, 2 n+1}^{(\lambda)}\right)}, \\
\int_{-1+1 / n^{2}}^{-1 / 2} \varphi^{(2 \lambda-1) m+(\lambda-1)}(t) d t \sim n^{(1-2 \lambda) m-1-\lambda}
\end{gathered}
$$

because $((2 \lambda-1) m+\lambda-1) / 2<(-3+\lambda) / 2<-1$. Therefore, we have the result.

\section{References}

[1] P. Vértesi, "Hermite-Fejér interpolations of higher order. II," Acta Mathematica Hungarica, vol. 54, no. 3-4, pp. 369-380, 1990.

[2] G. Szegö, "Über gewisse orthogonale polynome, die zu einer oszillierenden Belegungsfunktion gehören," Mathematische Annalen, vol. 110, no. 1, pp. 501-513, 1935.

[3] S. Ehrich and G. Mastroianni, "Stieltjes polynomials and lagrange interpolation," Mathematics of Computation, vol. 66, no. 217, pp. 311-331, 1997.

[4] S. Ehrich and G. Mastroianni, "On the generalized Stieltjes polynomials and Lagrange interpolation," in Approximation Theory and Function Series, vol. 5 of Bolyai Society Mathematical Studies, pp. 187-203, 1996.

[5] H. S. Jung, "Hermite and Hermite-Fejér interpolation for Stieltjes polynomials," Mathematics of Computation, vol. 75, no. 254, pp. 743-766, 2006.

[6] G. Szego, Orthogonal Polynomials, vol. 23, American Mathematical Society. Colloquium Publications, Providence, RI, USA, 1975.

[7] H. S. Jung and R. Sakai, "Asymptotic properties of derivatives of the Stieltjes Polynomials," Journal of Applied Mathematics, vol. 2012, Article ID 482935, 25 pages, 2012.
[8] P. Vértesi, "Hermite-Fejér interpolations of higher order. I," Acta Mathematica Hungarica, vol. 54, no. 1-2, pp. 135-152, 1989.

[9] Y. Kanjin and R. Sakai, "Pointwise convergence of HermiteFejér interpolation of higher order for Freud weights," Tohoku Mathematical Journal, vol. 46, no. 2, pp. 181-206, 1994.

[10] P. Nevai, "Mean convergence of lagrange interpolation, III," Transactions of the American Mathematical Society, vol. 282, pp. 669-698, 1984.

[11] S. Ehrich, "Asymptotic properties of Stieltjes polynomials and Gauss-Kronrod quadrature formulas," Journal of Approximation Theory, vol. 82, no. 2, pp. 287-303, 1995. 


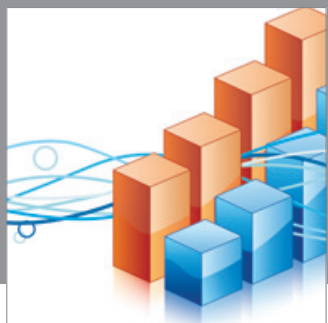

Advances in

Operations Research

mansans

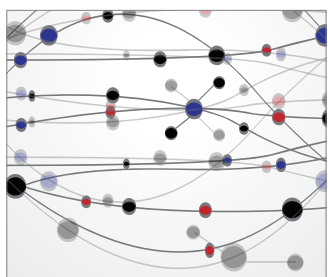

The Scientific World Journal
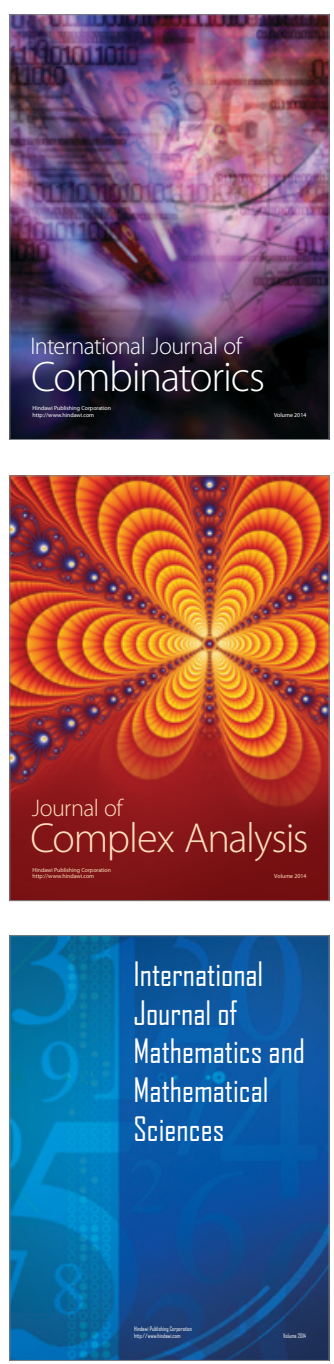
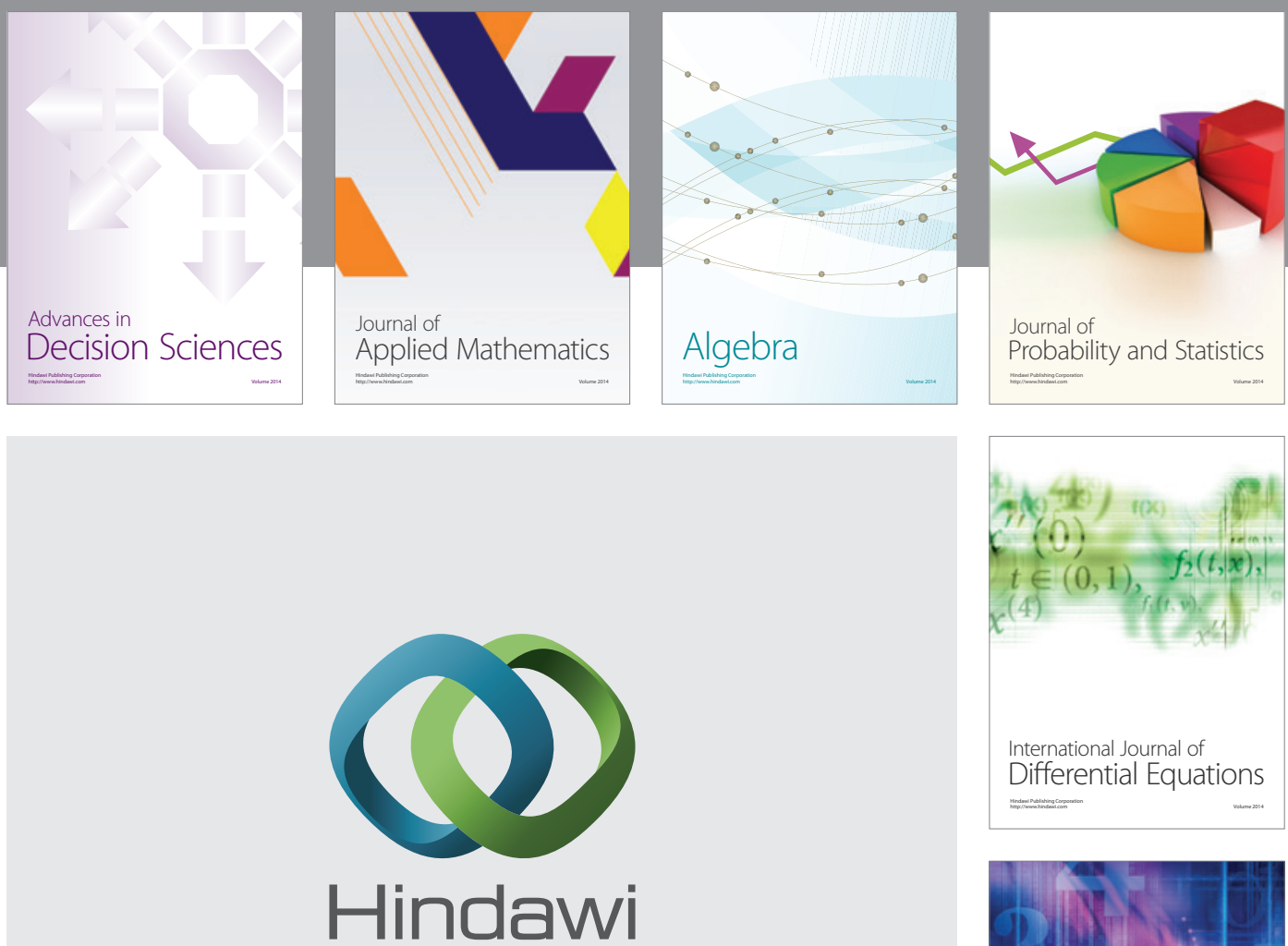

Submit your manuscripts at http://www.hindawi.com
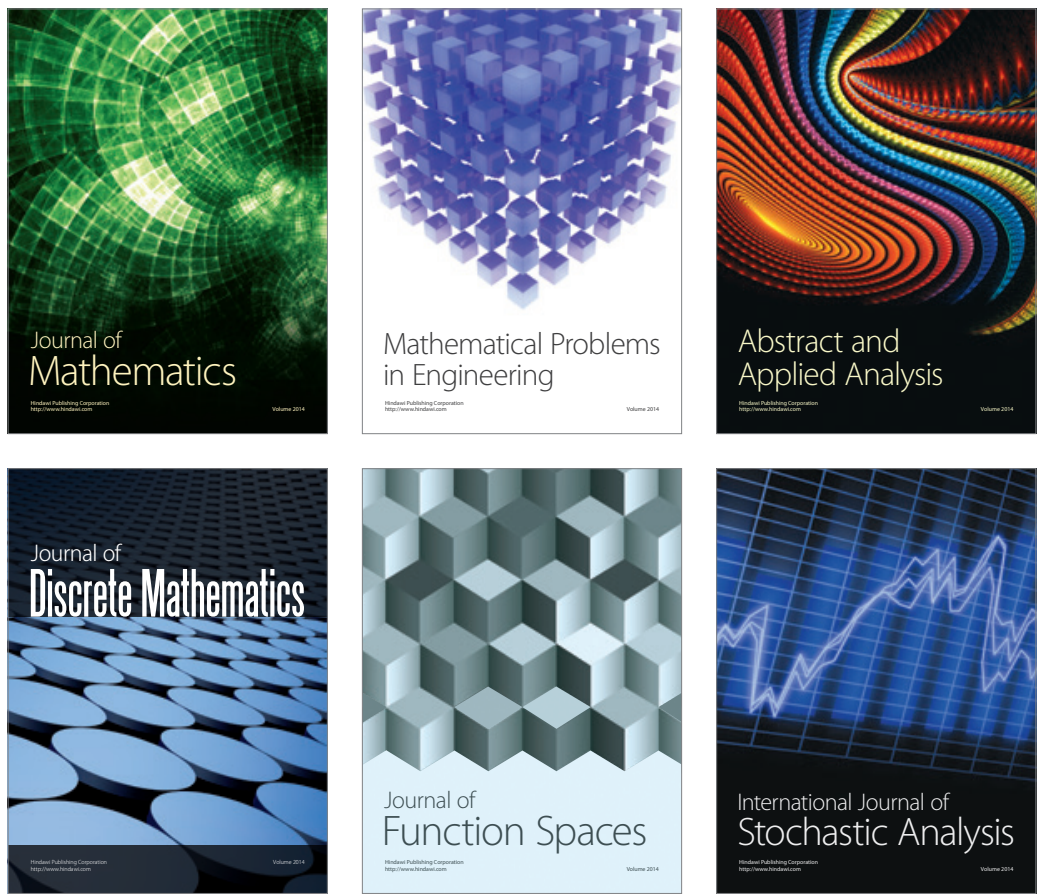

Journal of

Function Spaces

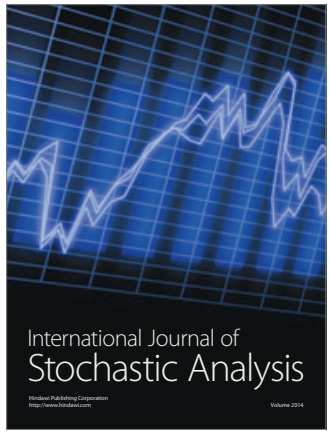

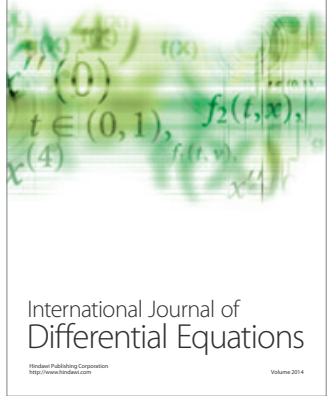
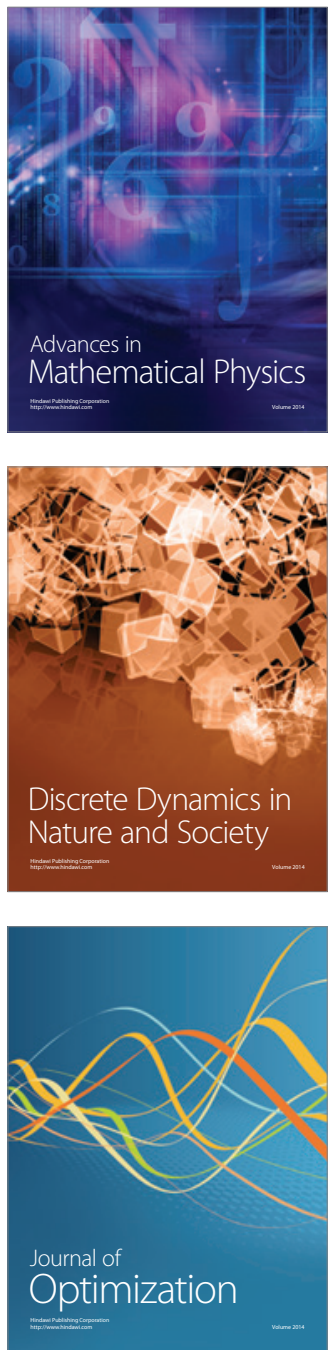